\title{
ROEDORES SIGMODONTÍNEOS (MAMMALIA, RODENTIA, CRICETIDAE) HOLOCÊNICOS DO RIO GRANDE DO SUL, BRASIL - O SÍTIO RS-TQ-58: AFONSO GARIVALDINO RODRIGUES
}

\author{
NARLA SHANNAY STUTZ, JORGE JOSÉ CHEREM \\ Departamento de Geociências, Universidade Federal de Santa Catarina, Campus Universitário, s/n, 88040-900, \\ Florianópolis, SC, Brasil.narla_stutz@yahoo.com.br,jjcherem@gmail.com \\ ULYSES FRANCISCO JOSÉ PARDIÑAS \\ Instituto de Diversidad y Evolución Austral (IDEAus-CONICET), CC 128, 9120 Puerto Madryn, Chubut, Argentina. \\ ulyses@cenpat-conicet.gob.ar \\ PATRÍCIA HADLER \\ Departamento de Geociências, UFSC, Campus Universitário, s/n, 88040-900, Florianópolis, SC, Brasil. \\ patricia.hadler@ufsc.br
}

\begin{abstract}
HOLOCENE SIGMODONTINE RODENTS (MAMMALIA, RODENTIA, CRICETIDAE) FROM RIO GRANDE DO SUL, BRAZIL - THE SITE RS-TQ-58: AFONSO GARIVALDINO RODRIGUES. We present the sigmodontine assemblage from lower and middle Holocene recorded in the archaeological site RS-TQ-58: Afonso Garivaldino Rodrigues (County of Brochier, State of Rio Grande do Sul). A total of 16 species are detected from the study of 572 specimens, about half of which belonging to the tribe Akodontini. The assemblage recorded include species which typically inhabit open areas (e.g. Calomys sp., Gyldenstolpia sp., Kunsia tomentosus) and also several considered as forest dewellers (e.g. Delomys sp., Juliomys sp., Sooretamys angouya). Necromys obscurus, a sigmodontine today restricted to Central Argentina and Southern Uruguay, is abundantly recorded in Garivaldino and reported for the first time to the Quaternary from Brazil. Thaptomys nigrita and Wilfredomys oenax have in Garivaldino their first records to the Holocene from Rio Grande do Sul State. Compared to the recent assemblage of sigmodontine rodents recorded in Rio Grande do Sul, Pseudoryzomys simplex, K. tomentosus, N. obscurus and Gyldenstolpia sp. became regionally extinct. The sigmodontine assemblage detected in Garivaldino is richest than a previously studied one from Pilger site. The evidence of both sites reflects changes in the species composition, which can be linked with a progressive transformation of the general landscape from mostly open grasslands to forests.
\end{abstract}

Key words: lower Holocene, Necromys obscurus, Sigmodontinae, Thaptomys nigrita, Wilfredomys oenax.

RESUMO - Apresentamos a assembleia de sigmodontíneos do Holoceno inferior e médio registrada no sítio arqueológico RS-TQ-58: Afonso Garivaldino Rodrigues (Município de Brochier, Estado do Rio Grande do Sul). Um total de 16 espécies foram detectadas pelo estudo de 572 espécimes, sendo quase metade destes pertencente a tribo Akodontini. Esta assembleia inclui espécies tipicamente de áreas abertas (e.g. Calomys sp., Gyldenstolpia sp., Kunsia tomentosus) e outras consideradas florestais (e.g. Delomys sp., Juliomys sp., Sooretamys angouya). Necromys obscurus, sigmodontíneo atualmente restrito à Argentina Central e sul do Uruguai, está presente de forma abundante no sítio Garivaldino e é registrado pela primeira vez para o Quaternário do Brasil. Thaptomys nigrita e Wilfredomys oenax tem seus primeiros registros para o Holoceno do Estado do Rio Grande do Sul. Em comparação com a assembleia atual de roedores sigmodontíneos conhecida para o Rio Grande do Sul, Pseudoryzomys simplex, K. tomentosus, N. obscurus e Gyldenstolpia sp. tornaram-se regionalmente extintos. A assembleia de sigmodontíneos registrada no Garivaldino é mais rica do que outra previamente estudada proveniente do sítio Pilger. A evidência de ambos os sítios reflete mudanças na composição de espécies, a qual pode estar relacionada com uma transformação progressiva da paisagem, de campos abertos para florestas.

Palavras-chave: Holoceno inferior, Necromys obscurus, Sigmodontinae, Thaptomys nigrita, Wilfredomys oenax. 


\section{INTRODUÇÃO}

Sigmodontinae constitui a subfamília dominante de roedores cricetídeos na América do Sul. Seus membros apresentam uma vasta gama de hábitos e características físicas, e ocupam diversos habitats, desde as planícies alagadas aos campos secos e dos ambientes costeiros aos Andes (Patton et al., 2015). Os primeiros registros fósseis de sigmodontíneos foram descritos por Peter Lund para a região de Lagoa Santa, Minas Gerais (e.g. Lund, 1840, 1950). No Brasil, estes ainda se constituem nos principais registros (cf. Winge, 1887), sendo o conhecimento sobre a ocorrência fóssil do grupo no país bastante escasso e exclusivo para o Quaternário.

Para o Holoceno do Rio Grande do Sul, escavações arqueológicas no nordeste do estado têm disponibilizado importantes coleções de mamíferos de pequeno porte. Esse material tem sido objeto de estudos recentes, especialmente os marsupiais e os roedores caviomorfos (e.g. Rodrigues, 2008; Hadler et al., 2008, 2009, 2016). Os roedores sigmodontíneos permanecem ainda pouco estudados, com apenas a assembleia do sítio RS-C-61: Adelar Pilger descrita detalhadamente quanto à taxonomia e considerações paleoambientais (Hadler et al., 2016).

Apesar da escassez de dados, foi possível propor uma hipótese inicial sobre a evolução ambiental holocênica do Rio Grande do Sul. Nesse sentido, um dos aspectos importantes acerca do registro de pequenos mamíferos até agora estudados indica que a assembleia de caviomorfos e sigmodontíneos durante o Holoceno inferior-médio estava dominada por espécies de ambiente aberto. Várias destas, incluindo o maior sigmodontíneo vivente (Kunsia tomentosus) e equimiídeos como Dicolpomys fossor e Clyomys riograndensis, desaparecem em direção ao Holoceno superior, enquanto se verifica um aumento de espécies de ambiente florestal. Estes processos são de grande relevância, já que implicam tanto em extinções de caráter regional como total, que poderiam estar relacionadas com mudanças ambientais que afetaram as principais formações vegetais (Hadler et al., 2013, 2016).

Neste contexto sobressai uma nova assembleia de sigmodontíneos escavada no sítio RS-TQ-58: Afonso Garivaldino Rodrigues (daqui em diante sítio Garivaldino), do Holoceno inferior e médio do Rio Grande do Sul. A composição taxonômica dessa assembleia é apresentada aqui e fornece novos dados sobre sigmodontíneos do Quaternário do Brasil.

\section{ÁREA DE ESTUDO}

O sítio Garivaldino localiza-se no Município de Brochier, no limite entre a Depressão Central e o Planalto da Serra Geral,

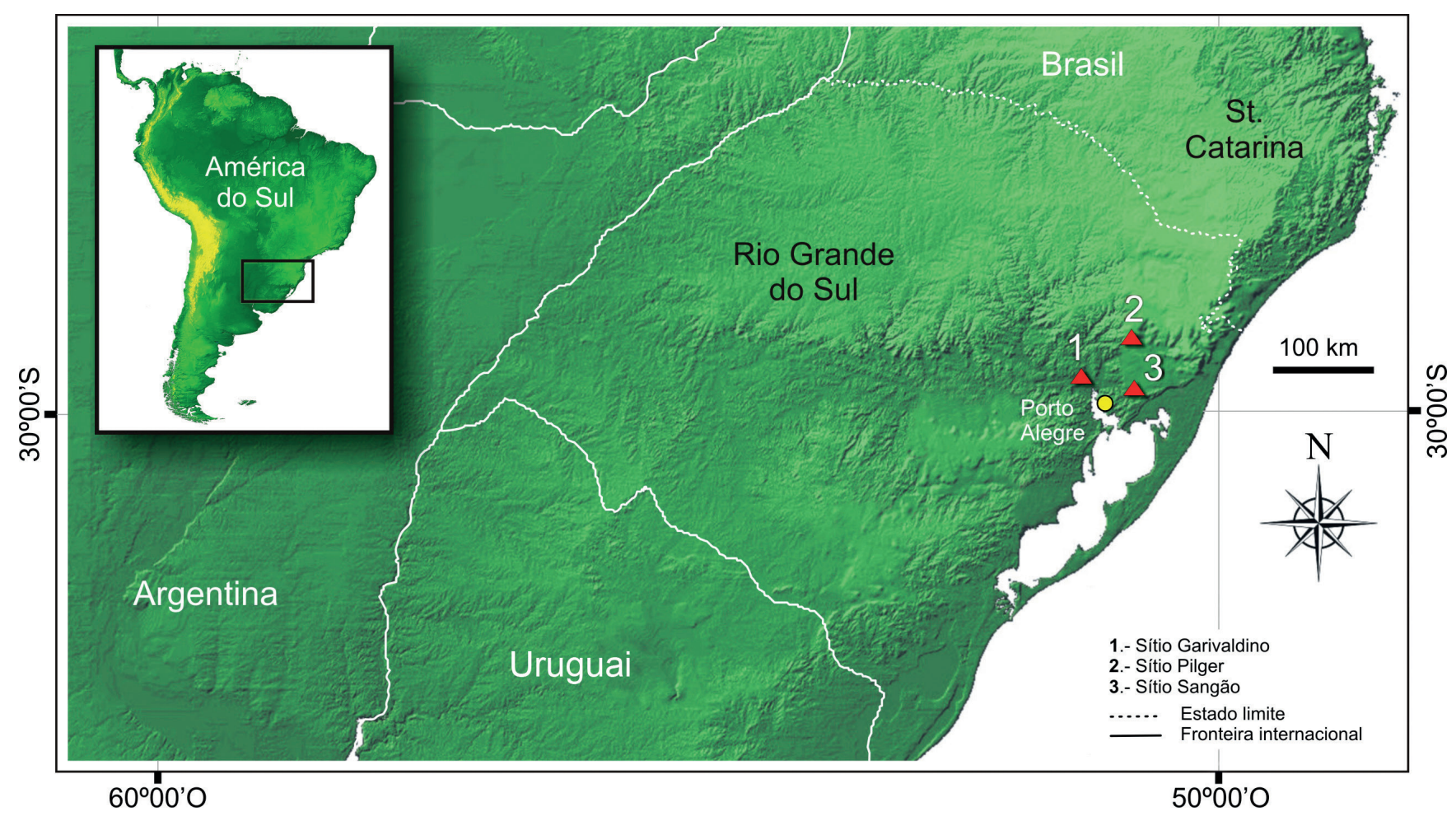

Figura 1. Mapa do Estado do Rio Grande do Sul (Brasil), indicando a localização dos sítios RS-C-61, Adelar Pilger; RS-TQ-58, Afonso Garivaldino Rodrigues e RS-S-327, Sangão.

Figure 1. Map of State of Rio Grande do Sul (Brazil), showing the location of RS-C-61, Adelar Pilger; RS-TQ-58, Afonso Garivaldino Rodrigues and RS-S-327, Sangão sites. 
Tabela 1. Idades radiocarbônicas para o sítio Garivaldino.

Table 1. Radiocarbon ages for the Garivaldino site.

\begin{tabular}{ccc}
\hline Idade (anos) & Profundidade $(\mathrm{cm})$ & Código \\
\hline $7250 \pm 350$ & $50-60$ & Beta 44740 \\
$8020 \pm 150$ & $130-140$ & Beta 33458 \\
$8290 \pm 130$ & $170-180$ & Beta 32183 \\
$9430 \pm 360$ & $200-210$ & Beta 4739 \\
\hline
\end{tabular}

sob as coordenadas $29^{\circ} 34^{\prime} 5^{\prime \prime} \mathrm{S}$ e $51^{\circ} 38^{\prime} 45^{\prime \prime} \mathrm{O}$ (Figura 1). O sítio é um abrigo sob rocha erodido no arenito da Formação Botucatu e está $72 \mathrm{~m}$ acima do nível do mar atual. Possui 21,40 $\mathrm{m}$ de abertura com direção norte, $8,50 \mathrm{~m}$ de profundidade $\mathrm{e}$ $8,60 \mathrm{~m}$ de altura (Mentz-Ribeiro \& Ribeiro,1999).

De acordo com Mentz-Ribeiro \& Ribeiro (1999), que apresentaram a planta baixa do abrigo, a escavação do sítio foi realizada em 12 quadrículas, com a utilização simultânea da técnica de superfícies amplas e a de níveis artificias de $10 \mathrm{~cm}$. Todo o sedimento retirado das quadrículas foi peneirado em malha de $0,1 \mathrm{~cm}$. Os achados arqueológicos incluem material lítico, cerâmica, e fragmentos de conchas e ossos, indicando que o sítio foi utilizado como habitação pelos caçadores-coletores da Tradição Umbu. O sítio Garivaldino apresenta as datações mais antigas conhecidas para os sítios arqueológicos em abrigos sob rocha no Estado do Rio Grande do Sul, abrangendo o Holoceno inferior e médio (Mentz-Ribeiro \& Ribeiro, 1999; Tabela 1).

\section{MATERIAL E MÉTODOS}

O material estudado está depositado no Centro de Ensino e Pesquisas Arqueológicas da Universidade de Santa Cruz do Sul (UNISC). Constitui-se de 572 fragmentos crâniomandibulares, com ou sem dentes incluídos de roedores sigmodontíneos, provenientes da quadrícula D6. Esta quadrícula foi escavada somente em níveis artificiais para servir como controle estratigráfico, totalizando 24 níveis.

O material foi limpo sob estereomicroscópio, com o auxílio de pincel e agulha, e numerado. A numeração de cada exemplar é composta por dois números, o primeiro indicando o nível no qual o material foi coletado e o segundo identificando o fragmento, seguindo uma ordem sequencial. A identificação foi realizada, sob estereomicroscópio, por comparação com espécimes recentes da Coleção de Mamíferos do Departamento de Ecologia e Zoologia da Universidade Federal de Santa Catarina (UFSC) e da Coleção de Mamíferos do Centro Nacional Patagónico (CNP, Puerto Madryn, Argentina), e com espécimes fósseis depositados na Seção de Arqueologia da Universidade Federal do Rio Grande do Sul (UFRGS), além de bibliografia (e.g. Hershkovitz, 1955; Voss \& Myers, 1991; Voss, 1993; Pardiñas, 1996; Pardiñas et al., 2008).
As medidas dos molares foram tomadas com paquímetro com precisão de $0,02 \mathrm{~mm}$. As descrições anatômicas seguiram os conceitos principais de Hershkovitz (1962) e Carleton \& Musser (1989). A nomenclatura das estruturas dentárias seguiu Reig (1977) e o protocolo de descrição Percequillo (2006). A sistemática dos roedores seguiu Patton et al. (2015) e Machado et al. (2015). O número mínimo de indivíduos (MNI) por táxon foi calculado de acordo com Beisaw (2013).

Abreviaturas: C, comprimento; L, largura; M1, M2, M3, primeiro, segundo e terceiro molares superiores; $\mathbf{m} 1, \mathbf{m} \mathbf{2}, \mathbf{m} 3$, primeiro, segundo e terceiro molares inferiores.

\section{SISTEMÁTICA}

Para o sítio Garivaldino, 34 fragmentos de maxilares e dentários foram identificados apenas ao nível de subfamília e os demais (538) representam 16 táxons, conforme descrito a seguir.

Ordem RODENTIA Bowdich, 1821 Subordem MYOMORPHA Brandt, 1855

Superfamília MUROIDEA Illiger, 1811

Família CRICETIDAE Fischer, 1817 Subfamília SIGMODONTINAE Wagner, 1843

Sigmodontinae gen. et sp. indet.

Material. Três fragmentos de maxilares e 31 fragmentos de dentários (Apêndice 1).

Descrição. $O$ estado fragmentário do material e o grande desgaste dos molares, ou a ausência destes, permitiram apenas atribuí-los à subfamília Sigmodontinae.

Sigmodontinae incertae sedis

Delomys Thomas, 1917

Espécie-tipo. Hesperomys dorsalis Hensel, 1872.

Delomys sp.

(Figuras 2A, D)

Material. 10 fragmentos de maxilares e 20 fragmentos de dentários (Apêndice 1).

Descrição. $\mathrm{O}$ maxilar e dentário possuem tamanho médio. Os molares superiores e inferiores são pentalofodontes e possuem as cúspides opostas umas às outras. $\mathrm{O}$ anterocone do M1 é largo, e possui flexo anteromediano profundo e deslocado lingualmente, sendo o cônulo anterolabial maior do que o anterolingual. O M1 e M2 possuem anterolofo e mesolofo desenvolvidos, sendo o último fusionado ao mesoestilo. $\mathrm{O} \mathrm{M} 2 \mathrm{e} \mathrm{M} 3$ possuem três raízes. $\mathrm{O} \mathrm{m} 1$ apresenta cíngulo anterolabial conspícuo. No m1 e m2 meso- e posterolofídeos são bem desenvolvidos. O ectolofídeo é bastante conspícuo e fusionado ao ectoestilídeo em $\mathrm{m} 1$ 

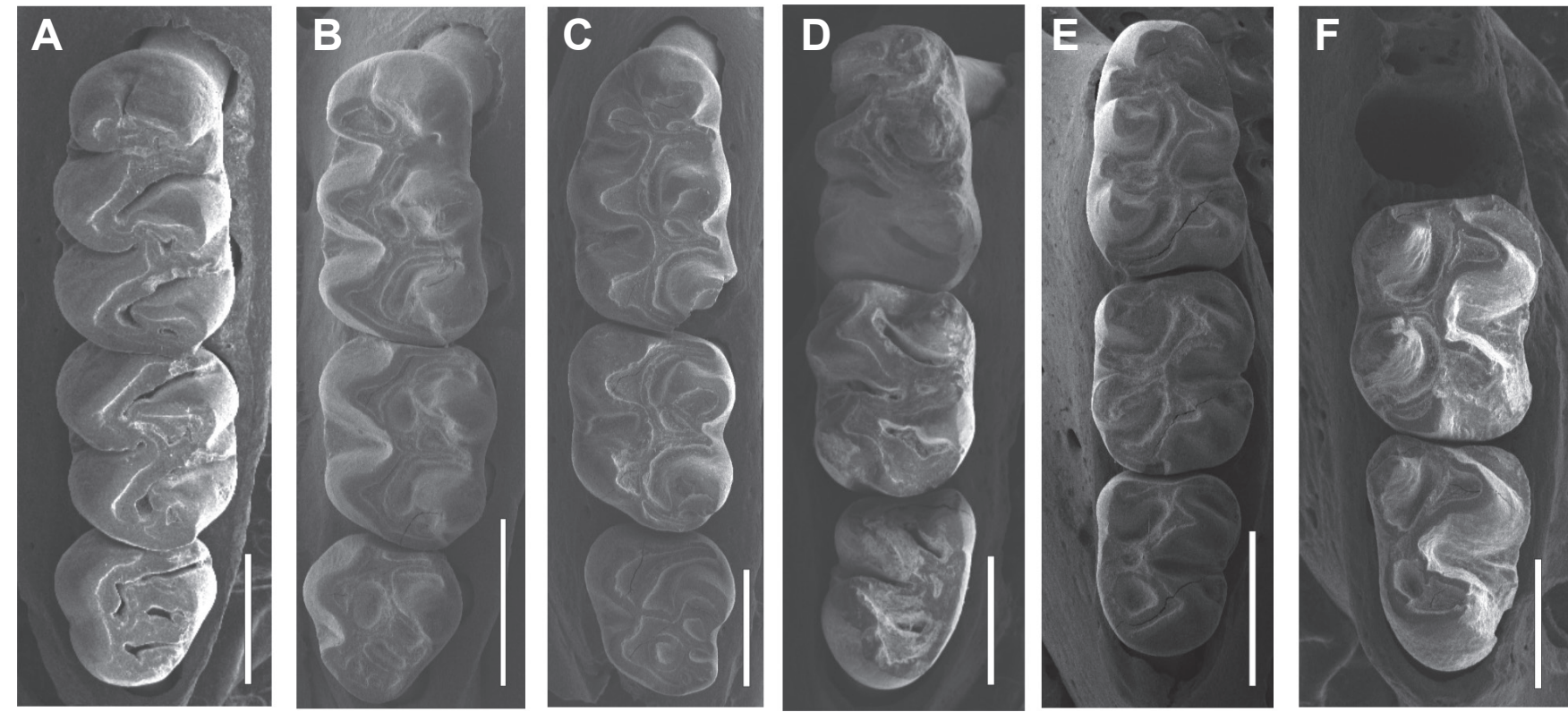

Figura 2. Vista oclusal dos molares superiores e inferiores de A, Delomys sp., UNISC 1753-305, M1-M3 esquerdo; B, Juliomys sp., UNISC 1753-303, M1-M3 esquerdo; C, Wilfredomys oenax, UNISC 1761-202, M1-M3 esquerdo; D, Delomys sp., UNISC 1752-212, m1-m3 esquerdo; E, Juliomys sp., UNISC 1754-209, m1-m3 direito; F, Wilfredomys oenax, UNISC 1757-215, m2-m3 direito. Escalas = $1 \mathrm{~mm}$.

Figure 2. Occlusal view of upper and lower molars of A, Delomys sp., UNISC 1753-305, left M1-M3; B, Juliomys sp., UNISC 1753-303, left M1-M3; C, Wilfredomys oenax, UNISC 1761-202, left M1-M3; D, Delomys sp., UNISC 1752-212, left m1-m3; E, Juliomys sp., UNISC 1754209, right m1-m3; F, Wilfredomys oenax, UNISC 1757-215, right $\mathrm{m} 2-\mathrm{m} 3$. Scale bars $=1 \mathrm{~mm}$

e em alguns $m 2$, enquanto em outros $m 2$ há somente $\mathrm{o}$ ectoestilídeo, sem ectolofídeo.

Comentários. As características descritas acima para o material do sítio Garivaldino permitiram atribuí-lo ao gênero Delomys, de acordo com Voss (1993). Este gênero inclui atualmente três espécies: D. altimontanus Gonçalves \& Oliveira, 2014, D. dorsalis (Hensel, 1872) e D. sublineatus (Thomas, 1903), sendo que somente a segunda tem registro para o Rio Grande do Sul (Gonçalves \& Oliveira, 2014). As espécies de Delomys são diferenciadas principalmente por características da pelagem, métricas e genéticas (Voss, 1993; Gonçalves \& Oliveira, 2014). Neste contexto, não é possível a atribuição específica do material fóssil (e.g. ver discussão sobre o status de Calomys plebejus Winge, 1887 em Voss, 1993).

Juliomys González, 2000

Espécie-tipo. Thomasomys pictipes Osgood, 1933.

Juliomys sp.

(Figuras 2B, E)

Material. 16 fragmentos de maxilares e 53 fragmentos de dentários (Apêndice 1).

Descrição. O palato é curto. No dentário, a projeção capsular é bem desenvolvida. Os molares superiores e inferiores são pentalofodontes e possuem a topografia coronal cristada. As séries dentárias são divergentes posteriormente. $\mathrm{O}$ anterocone é largo e possui um flexo anteromediano profundo, sendo o cônulo anterolabial maior do que o anterolingual. O M1 e M2 apresentam mesolofo bem desenvolvido. O M3 possui flexos rasos e tem as cúspides posteriores pouco desenvolvidas. $\mathrm{O} \mathrm{m} 1$ possui anteroconídeo mais largo e um flexídeo anteromediano mais profundo do que os espécimes identificados como Oligoryzomys sp. No m1 e m2 o mesolofídeo é independente, bem desenvolvido e fusionado ao mesoestilídeo, e o ectolofídeo é desenvolvido e fusionado ao ectoestilídeo. Nos exemplares com maior desgaste nos molares, o mesolofídeo e o ectolofídeo tornam-se menos visíveis.

Comentários. As características listadas acima permitiram identificar o material do sítio Garivaldino apenas ao nível de gênero (González, 2000; González et al., 2015b; Christoff et al., 2016). As dimensões dos espécimes fósseis e o grau de desenvolvimento do ectolofídeo sugerem que eles possam se referir a Juliomys ossitenuis Costa, Pavan, Leite \& Fagundes, 2007 ou a J. ximenezi Christoff, Vieira, Oliveira, Gonçalves, Valiati \& Tomasi, 2016, ambas atualmente presentes no Rio Grande do Sul (Costa et al., 2007; Christoff et al., 2016).

Tribo AKODONTINI Vorontsov, 1959

Akodon Meyen, 1833

Espécie-tipo. Akodon boliviense Meyen, 1833.

Akodon sp.

(Figuras 3A, 4A) 

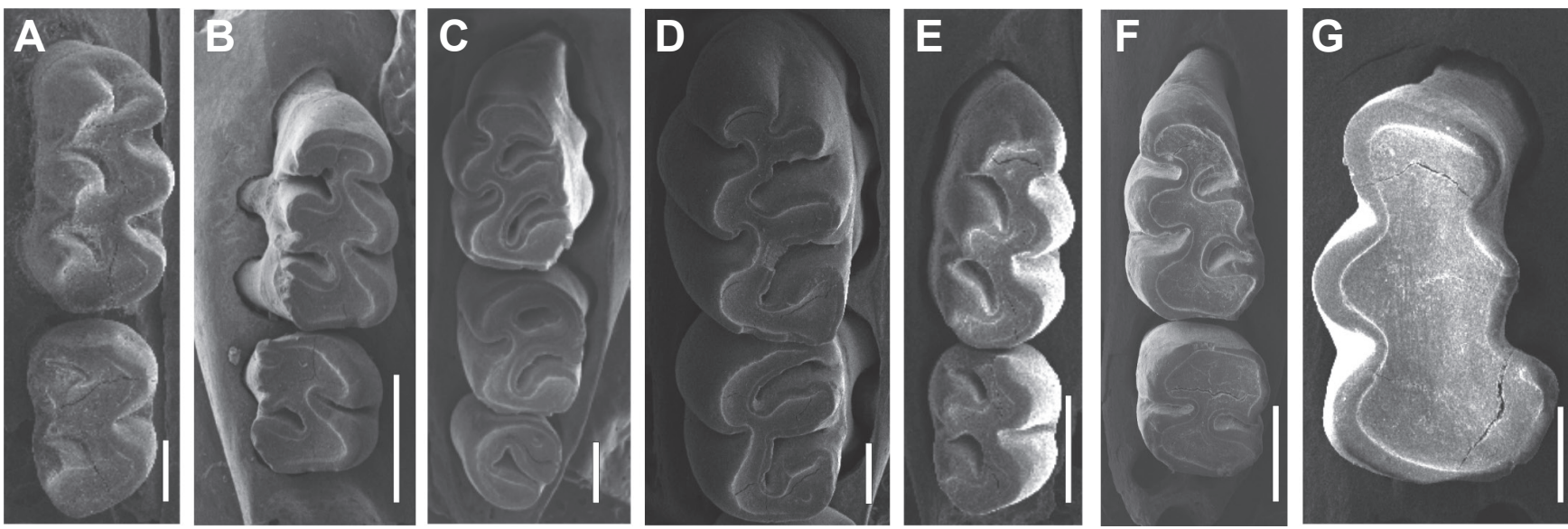

Figura 3. Vista oclusal dos molares superiores dos Akodontini. A, Akodon sp., UNISC 1753-301, M1-M2 direito; B, Bibimys labiosus, UNISC 1758-207, M1-M2 direito; C, Gyldenstolpia sp., UNISC 1770-203, M1-M3 esquerdo; D, Kunsia tomentosus, UNISC 1757-200, M1-M2 esquerdo; E, Necromys lasiurus, UNISC 1753-321, M1-M2 direito; F, Necromys obscurus, UNISC 1757-262, M1-M2 esquerdo; G, Oxymycterus sp. UNISC 1757-257, M1 esquerdo. Escalas: A, G = 0,5 mm; B-F = $1 \mathrm{~mm}$.

Figure 3. Occlusal view of upper molars of Akodontini. A, Akodon sp., UNISC 1753-301, right M1-M2; B, Bibimys labiosus, UNISC 1758-207, M1-M2 right; C, Gyldenstolpia sp., UNISC 1770-203, left M1-M3; D, Kunsia tomentosus, UNISC 1757-200, left M1-M2; E, Necromys lasiurus, UNISC 1753-321, right M1-M2; F, Necromys obscurus, UNISC 1757-262, left M1-M2; G, Oxymycterus sp., UNISC 1757-257, left M1. Scale bars: $A, G=0,5 \mathrm{~mm}, \mathrm{~B}-\mathrm{F}=1 \mathrm{~mm}$.

Material. Seis fragmentos de maxilares e 95 fragmentos de dentários (Apêndice 1).

Descrição. O forame incisivo é longo, estendendose posteriormente até o nível do protocone do M1. O dentário é delicado e baixo. Os molares superiores e inferiores possuem cúspides levemente alternadas. Os molares superiores possuem mesolofo. O M1 apresenta flexo anteromediano profundo. O M3 é cilíndrico. Os molares inferiores apresentam mesolofídeo moderado. No $\mathrm{m} 1$, o flexídeo anteromediano é profundo e centralizado, enquanto em Necromys lasiurus é geralmente ausente, e o protoestilídeo está presente. $\mathrm{O} \mathrm{m} 2$ e $\mathrm{m} 3$ possuem protoflexídeo. $\mathrm{O} \mathrm{m} 3$ possui formato sigmoide e é quase tão longo quanto o $\mathrm{m} 2$.

Comentários. Essas características permitiram assinalar o material ao gênero Akodon, de acordo com Reig (1972) e Pardiñas et al. (2015b). Esse material seguramente engloba dois morfótipos, que podem corresponder a A. montensis Thomas, 1913 e A. paranaensis Christoff, Fagundes, Sbalqueiro, Mattevi \& Yonenaga-Yassuda, 2000. No entanto, as espécies de Akodon, em especial do grupo cursor (cf. Smith \& Patton, 2007), que ocorrem no Brasil, são muito similares morfologicamente (Christoff et al., 2000; Pardiñas et al. 2015b). Desta forma, optou-se por não atribuir nomes específicos aos espécimes fósseis.

Bibimys Massoia, 1979

Espécie-tipo. Bibimys torresi Massoia, 1979.

Bibimys labiosus (Winge, 1887)

(Figuras 3B, 4B)
Material. Um fragmento de maxilar e 11 fragmentos de dentários (Apêndice 1).

Descrição. O dentário é pequeno, alto e robusto. A projeção capsular é pouco desenvolvida e na porção interna do côndilo está presente uma depressão profunda, em formato de "colher". No M1 e M2 o mesolofo está fusionado ao paralófulo. O M1 possui quatro raízes e o M2, três. Os molares inferiores apresentam distoflexídeo (sensu Hershkovitz, 1990; ver Pardiñas, 1996) bem marcado. O flexídeo anteromediano é deslocado lingualmente. $\mathrm{O} \mathrm{m} 1$, quando com menor desgaste, apresenta metalofulídeo. O $\mathrm{m} 3$ possui hipoflexídeo bem desenvolvido. $\mathrm{O} \mathrm{m} 1$ possui quatro raízes e o $\mathrm{m} 2$ e o $\mathrm{m} 3$ possuem três raízes.

Comentários. As características descritas permitiram atribuir o material ao gênero Bibimys, de acordo com Pardiñas (1996). Três espécies são atualmente reconhecidas, B. chacoensis (Shamel, 1931), B. labiosus e B. torresi Massoia, 1979, porém os limites entre elas são tênues e parecem estar relacionados principalmente à escassez de espécimes coletados (Pardiñas, 1996; D’Elía et al., 2006). As diferenças morfológicas entre B. labiosus e B. chacoensis, por exemplo, não parecem ser significativas; e com o aumento de localidades conhecidas para a primeira, as áreas de distribuição de ambas as espécies parecem ser contínuas, incluindo desde o Brasil até o Paraguai e Argentina (Pardiñas et al., 2015a). Uma hipótese de trabalho seria considerar $B$. chacoensis sinônimo júnior de $B$. labiosus. Em função disto e pelo fato de $B$. labiosus ser a única espécie que ocorre atualmente no Brasil, o material do sítio Garivaldino foi identificado como B. labiosus. 

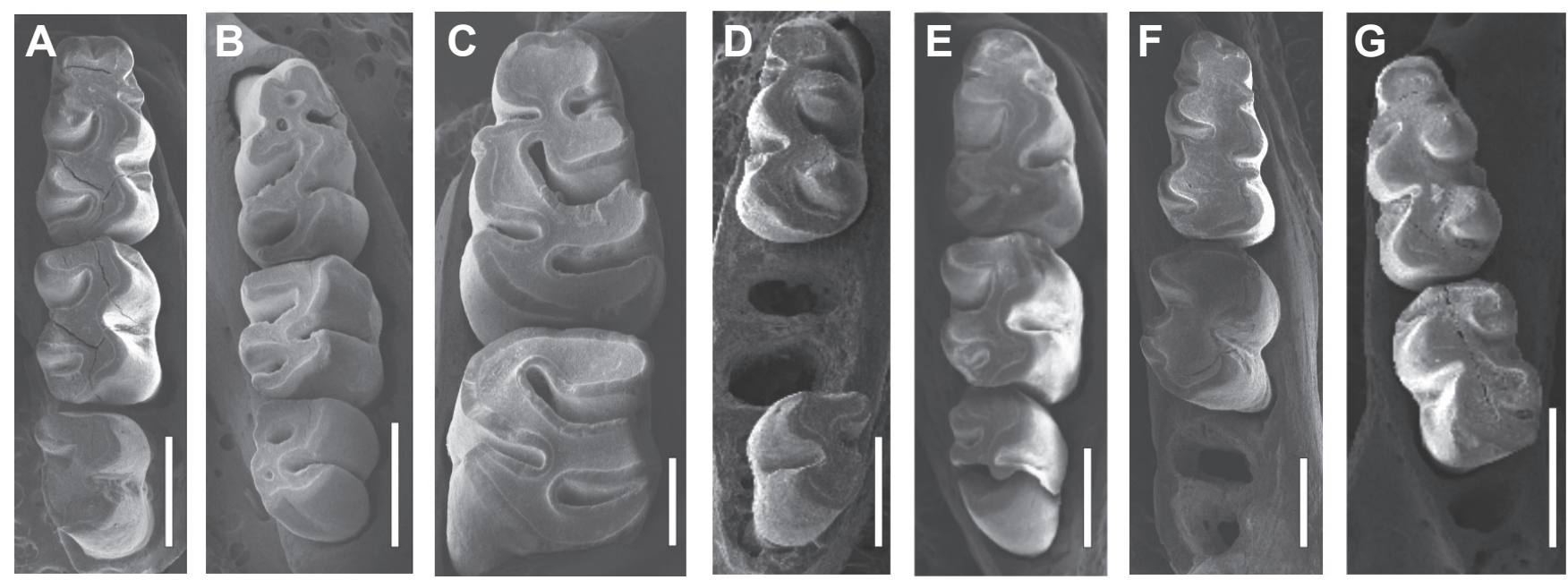

Figura 4. Vista oclusal dos molares inferiores dos Akodontini. A, Akodon sp., UNISC 1753-274, m1-m3 direito; B, Bibimys labiosus, UNISC 1752-213, m1-m3 direito; C, Gyldenstolpia sp., UNISC 1765-205, m1-m2 esquerdo; D, Necromys lasiurus, UNISC 1754-228, m1 e m3 esquerdo; E, Necromys obscurus, UNISC 1754-224, m1-m3 direito; F, Oxymycterus sp., UNISC 1765-206, m1-m2 direito; G, Thaptomys nigrita, UNISC 1752-231, $\mathrm{m} 1-\mathrm{m} 2$ esquerdo. Escalas $=1 \mathrm{~mm}$.

Figure 4. Occlusal view of lower molars of Akodontini. A, Akodon sp., UNISC 1753-274, right m1-m3; B, Bibimys labiosus, UNISC 1752-213, right m1-m3; C, Gyldenstolpia sp., UNISC 1765-205, left m1-m2; D, Necromys lasiurus, UNISC 1754-228, left m1 and m3; E, Necromys obscurus, UNISC 1754-224, right m1-m3; F, Oxymycterus sp., UNISC 1765-206, right m1-m2; G, Thaptomys nigrita, UNISC 1752-231, left $\mathrm{m} 1-\mathrm{m} 2$. Scale bars $=1 \mathrm{~mm}$.

Espécie-tipo. †Scapteromys fronto Winge, 1887.

\section{Gyldenstolpia sp.}

(Figuras 3C, 4C)

Material. Cinco fragmentos de maxilares e 11 fragmentos de dentários (Apêndice 1).

Descrição. Os molares superiores e inferiores são grandes (CM1-M3 UNISC 1765-200 = 7,86 mm; UNISC 1770-203 $=7,22 \mathrm{~mm} ; \mathrm{Cm} 1-\mathrm{m} 3$ UNISC $1756-200=8,44 \mathrm{~mm} ;$ UNISC $1765-205=8,4 \mathrm{~mm}$ e UNISC $1766-200=8,1 \mathrm{~mm})$. O M1 possui anterocone indiviso e o M3 é cilíndrico. Nos molares inferiores o mesolofídeo é bem desenvolvido. $\mathrm{O} \mathrm{m} 1$ apresenta protoconulídeo associado ao protoconídeo e possui três raízes. Comentários. As características descritas acima e a ausência de mesolofo nos molares superiores permitiram atribuir o material ao gênero Gyldenstolpia de acordo com Pardiñas et al. (2008). No entanto, conforme estes autores, as diferenças entre as duas espécies do gênero são sutis, optando-se aqui pela taxonomia aberta.

\section{Kunsia Hershkovitz, 1966}

Espécie-tipo. Mus tomentosus Lichtenstein, 1830.

Kunsia tomentosus (Lichtenstein, 1830)

(Figura 3D)

Material. Quatro fragmentos de maxilares e um fragmento de dentário (Apêndice 1).

Descrição. O dentário é alto, robusto e possui quatro alvéolos para o $\mathrm{m} 1$ e três alvéolos para o $\mathrm{m} 2$ e $\mathrm{m} 3$. Os molares superiores são grandes (CM1-M3 UNISC $1757-$ $200=10,3 \mathrm{~mm}$ ), planos e trilofodontes. O anterocone do M1 possui flexo anteromediano. O M3 é cilíndrico e possui três raízes.

Comentários. As características listadas acima e a ausência de mesolofo nos molares superiores permitiram a atribuição do material a $K$. tomentosus, de acordo com Pardiñas et al. (2008) e Bezerra \& Pardiñas (2016).

Necromys Ameghino, 1889

Espécie-tipo. $\dagger$ Necromys conifer Ameghino, 1889.

\section{Necromys lasiurus}

(Figuras 3E, 4D)

Material. Dezessete fragmentos de maxilares e 48 fragmentos de dentários (Apêndice 1).

Descrição. Os molares superiores e inferiores são tetralofodontes, com cúspides levemente alternadas. Os molares superiores possuem lofos transversais. $\mathrm{O}$ anterocone do M1 é indiviso. O M1 e M2 apresentam mesoestilo. O M2 possui protoflexo muito pouco marcado. $\mathrm{O}$ anteroconídeo do $\mathrm{m} 1$ é indiviso na maioria dos exemplares. $\mathrm{O} \mathrm{m} 1 \mathrm{e} \mathrm{m} 2$ possuem hipoflexídeo desenvolvido e ectoestilídeo conectado a um ectolofídeo pequeno em alguns exemplares.

Comentários. Essas características e a ausência de enteroestilo, enterolofo e mesolofídeo permitiram atribuir o material estudado ao gênero Necromys, conforme Reig (1972) e Anderson \& Olds (1989). Em comparação com $N$. obscurus, esse material foi identificado como $N$. lasiurus por 
Tabela 2. Medidas (mm) dos molares inferiores e série dentária de Necromys obscurus. Abreviações: C, comprimento; L, largura.

Table 2. Measurements (mm) of the lower molars and dental series of Necromys obscurus. Abbreviations: C, length; L, width.

\begin{tabular}{|c|c|c|c|c|c|c|c|}
\hline Espécime & $\mathrm{Cm} 1$ & Lm1 & $\mathrm{Cm} 2$ & $\mathrm{Lm} 2$ & $\mathrm{Cm} 3$ & Lm3 & $\mathrm{Cm} 1-\mathrm{m} 3$ \\
\hline UNISC 1752-208 & 2,24 & 1,32 & 1,68 & 1,36 & & & \\
\hline UNISC 1753-202 & 2,02 & 1,16 & 1,26 & 1,2 & & & \\
\hline UNISC 1753-214 & 2,04 & 1,2 & 1,46 & 1,27 & & & \\
\hline UNISC $1753-220$ & 2,36 & 1,29 & & & & & \\
\hline UNISC 1753-231 & 2,21 & 1,19 & & & & & \\
\hline UNISC 1753-277 & 2,16 & 1,2 & 1,68 & 1,38 & & & \\
\hline UNISC 1754-217 & 2,12 & 1,27 & 1,57 & 1,29 & & & \\
\hline UNISC 1754-224 & 2 & 1,19 & 1,38 & 1,3 & 1,15 & 1,02 & 5,11 \\
\hline UNISC 1756-202 & 2,06 & 1,2 & & & & & \\
\hline UNISC 1756-205 & 2,25 & 1,27 & 1,66 & 1,38 & & & \\
\hline UNISC 1756-208 & 2,24 & 1,19 & 1,52 & 1,32 & & & \\
\hline UNISC 1756-222 & & & 1,69 & 1,2 & & & \\
\hline UNISC 1756-226 & 2,17 & 1,24 & 1,53 & 1,37 & & & \\
\hline UNISC 1757-203 & & & 1,55 & 1,26 & & & \\
\hline UNISC 1757-204 & 2,2 & 1,34 & 1,7 & 1,39 & & & \\
\hline UNISC 1757-207 & 2,17 & 1,2 & & & & & \\
\hline UNISC 1757-221 & 2,2 & 1,17 & 1,5 & 1,14 & & & \\
\hline UNISC 1757-226 & 1,98 & 1,12 & & & & & \\
\hline UNISC 1757-235 & & & 1,59 & 1,3 & & & \\
\hline UNISC $1757-240$ & 2,27 & 1,31 & & & & & \\
\hline UNISC 1758-214 & 2,31 & 1,38 & & & & & \\
\hline UNISC $1758-220$ & 2,3 & 1,21 & 1,71 & 1,37 & & & \\
\hline UNISC 1759-211 & 2,26 & 1,33 & 1,59 & 1,38 & & & \\
\hline UNISC $1760-200$ & & & 1,67 & 1,28 & & & \\
\hline
\end{tabular}

apresentar maxilares e dentários gráceis, e dentes pequenos, como observado em espécimes recentes desta espécie (e.g. UFSC 3599, 3600, 3876, 4714, 4815).

Necromys obscurus (Waterhouse, 1837)

(Figuras 3F, 4E)

Material. Seis fragmentos de maxilares e 31 fragmentos de dentários (Apêndice 1).

Descrição. Os maxilares são robustos. Os dentários possuem tamanho médio, e são robustos e altos. O processo coronoide é robusto e voltado posteriormente. A projeção capsular é conspícua e situada de forma centralizada entre os processos coronoide e condiloide. A crista massetérica é mais desenvolvida do que nos espécimes de Necromys lasiurus do sítio Garivaldino. Os molares superiores são largos, e possuem cúspides opostas umas às outras. O M1 possui o anterocone em forma de leque e o anterolofo desenvolvido e projetado posteriormente. O M1 e M2 apresentam mesolofo desenvolvido e conectado ao paralófulo. Os molares inferiores possuem as cúspides levemente alternadas. $\mathrm{O} \mathrm{m} 1$ possui cíngulo anterolabial e um ectolofídeo pequeno. $\mathrm{O} \mathrm{m} 2$ possui protoflexídeo raso, menos marcado do que em $N$. lasiurus.

Comentários. As medidas do material (Tabela 2), o processo coronoide robusto e voltado posteriormente, a projeção capsular conspícua e centralizada entre os processos condiloide e coronoide e anterocone do M1 em forma de leque são características que permitiram atribuir esses espécimes a Necromys obscurus, de acordo com Galliari \& Pardiñas (2000). Atualmente $N$. obscurus habita áreas do sul do Uruguai e uma pequena área do centro-leste da Argentina, não ocorrendo no Brasil. No entanto, de acordo com Pardiñas et al. (2015c), N. obscurus teria tido uma distribuição mais ampla no passado, ocupando uma porção importante do território da Província de Buenos Aires (Galliari \& Pardiñas, 2000).

Cabe registrar aqui que os espécimes UFRGS 129-24', 133-2', 133-6', 135-52', 136-13', 137-14', 137-49', 157-1', 

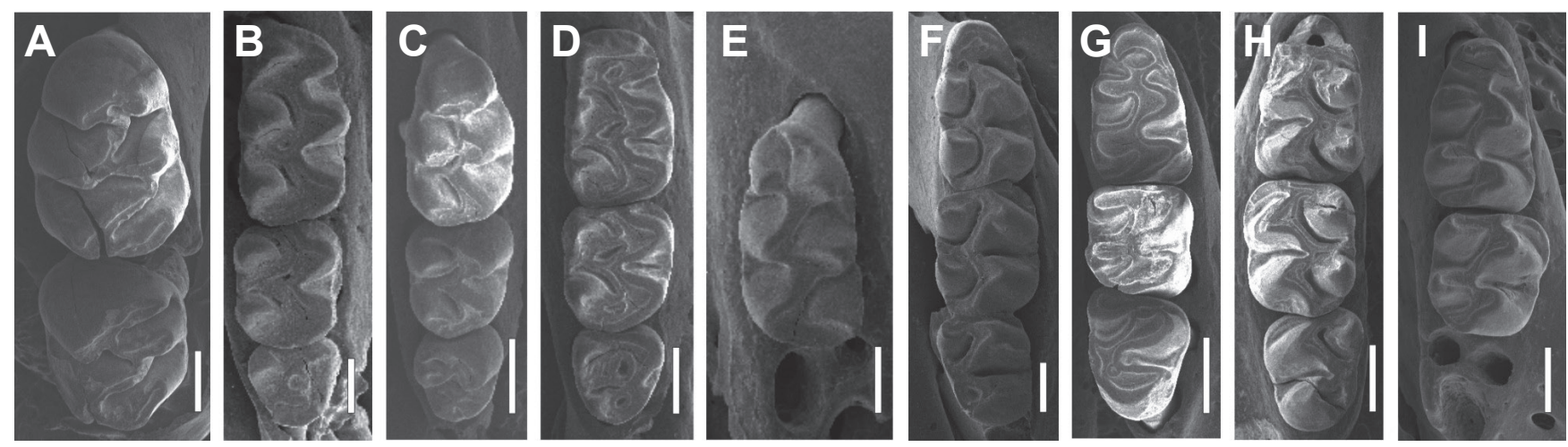

Figura 5. Vista oclusal dos molares superiores e inferiores dos Oryzomyini e Phyllotini. A, Holochilus cf. H. brasiliensis, UNISC 1758-200, M1-M2 esquerdo; B, Oligoryzomys sp., UNISC 1752-205, M1-M3 direito; C, Pseudoryzomys simplex, UNISC 1761-201, M1-M3 direito; D, Sooretamys angouya, UNISC 1752-200, M1-M3 direito; E, Calomys sp., UNISC 1757-263, M1 esquerdo; F, Oligoryzomys sp., UNISC 1753-247, m1-m3 direito; G, Pseudoryzomys simplex, UNISC 1752-216, m1-m3 direito; H, Sooretamys angouya, UNISC 1758-219, m1-m3 esquerdo; I, Calomys sp., UNISC 1753-295, m1-m2 direito. Escalas: A, C, D, G, H=1 mm; B, E, F, I = 0,5 mm.

Figure 5. Occlusal view of upper and lower molars of Oryzomyini and Phyllotini. A, Holochilus cf. H. brasiliensis, UNISC 1758-200, left M1-M2; B, Oligoryzomys sp., UNISC 1752-205, right M1-M3; C, Pseudoryzomys simplex, UNISC 1761-201, right M1-M3; D, Sooretamys angouya, UNISC 1752-200, right M1-M3; E, Calomys sp., UNISC 1757-263, left M1; F, Oligoryzomys sp., UNISC 1753-247, right m1-m3; G, Pseudoryzomys simplex, UNISC 1752-216, right m1-m3; H, Sooretamys angouya, UNISC 1758-219, left m1-m3; I, Calomys sp., UNISC 1753-295, right m1-m2. Scale bars: $A, C, D, G, H=1 \mathrm{~mm} ; B, E, F, I=0,5 \mathrm{~mm}$.

$157-17^{\prime}, 158-06^{\prime}, 159-15^{\prime}, 159-34^{\prime}, 159-43^{\prime}, 160-43^{\prime}$ do sítio Pilger, nordeste do Rio Grande do Sul, identificados como Necromys cf. N. lasiurus por Hadler et al. (2016), referem-se a $N$. obscurus. Da mesma forma, UFRGS 16032 ', atribuído a Scapteromys meridionalis por esses autores (Hadler et al., 2016: Figura 4I), representa um juvenil de N. obscurus.

Oxymycterus Waterhouse, 1837

Espécie-tipo. Mus nasutus Waterhouse, 1837.

Oxymycterus sp.

(Figuras 3G, 4F)

Material. Cinco fragmentos de maxilares e 17 fragmentos de dentários (Apêndice 1).

Descrição. O dentário é baixo e alongado. A crista massetérica e a projeção capsular são muito pouco desenvolvidas e o processo coronoide é relativamente baixo. Os molares superiores e inferiores são alongados, estreitos e tetralofodontes. O M1 apresenta flexo anteromediano. O m1 e $\mathrm{m} 2$ apresentam posterolofídeo bem desenvolvido.

Comentários. As características acima permitiram identificar o material apenas a nível de gênero, conforme Hinojosa et al. (1987) e Hershkovitz (1994). Um espécime do sítio Garivaldino (UNISC 1753-291) apresenta a série molar inferior completa e seu comprimento $(\mathrm{Cm} 1-\mathrm{m} 3=5,68 \mathrm{~mm})$ indica tratar-se de uma espécie de grande porte, conforme comparação com medidas de espécimes recentes de Oxymycterus quaestor Thomas, $1903(\mathrm{Cm} 1-\mathrm{m} 3=$ de 5,44 a $6,08 \mathrm{~mm})$ e $O$. nasutus (Waterhouse, 1837) $(\mathrm{Cm} 1-\mathrm{m} 3=\mathrm{de}$ $4,6$ a $4,84 \mathrm{~mm})$.
Thaptomys Thomas, 1916

Espécie-tipo. Hesperomys subterraneus Hensel, 1873 (= Mus nigrita Lichtenstein, 1829).

Thaptomys nigrita (Lichtenstein, 1829)

(Figura 4G)

Material. Três fragmentos de dentários (Apêndice 1).

Descrição. O forame mentoniano é grande e visível lateralmente. Os dentes são muito pequenos com as cúspides oblíquas. $\mathrm{O} \mathrm{m} 1$ possui flexídeo anteromediano bem desenvolvido e cíngulo anterolabial conspícuo. $\mathrm{O} \mathrm{m} 1$ e m2 possuem pequeno mesoestilídeo, e ectoestilídeo e posterolofídeo marcados. O m3 é bilofodonte, não possuindo posteroflexídeo.

Comentários. Essas características permitiram atribuir o material a Thaptomys nigrita, de acordo com Hershkovitz (1998).

Tribo ORYZOMYINI Vorontsov, 1959

Holochilus Brandt, 1835

Espécie-tipo. Holochilus sciureus Wagner, 1842.

Holochilus cf. H. brasiliensis (Desmarest, 1819)

(Figura 5A)

Material. Dois fragmentos de maxilares (Apêndice 1).

Descrição. Os molares superiores são grandes (UNISC 1758- 200: $\mathrm{CM} 1=3,38 \mathrm{~mm} ; \mathrm{CM} 2=2,18 \mathrm{~mm}$; UNISC 1758 204: $\mathrm{CM} 3=2,66 \mathrm{~mm}$ ), hipsodontes e com coroas aplainadas. 
Tabela 3. Medidas $(\mathrm{mm})$ das séries molares superior e inferior de Oligoryzomys sp. Abreviação: C, comprimento.

Table 3. Measurements $(\mathrm{mm})$ of the upper and lower molar series of Oligoryzomys sp. Abreviation: C, length.

\begin{tabular}{ccc}
\hline Espécime & CM1-M3 & Cm1-m3 \\
\hline UNISC 1752-205 & 3,68 & \\
UNISC 1753-318 & 3,84 & \\
UNISC 1755-244 & 3,72 & \\
UNISC 1753-227 & & 3,68 \\
UNISC 1753-247 & & 4,10 \\
UNISC 1755-220 & 3,60 \\
UNISC 1755-234 & & 3,70 \\
UNISC 1757-248 & & 3,72 \\
UNISC 1763-200 & & 3,84 \\
\hline
\end{tabular}

As cúspides são arredondadas e opostas umas às outras. O M1 e M2 possuem mesolofo vestigial. O M3 é grande, com hipoflexo e paraflexo profundos e opostos. O M1 possui quatro raízes.

Comentários. As características listadas acima, em particular a presença de mesolofo no M1 e no M2 e de cúspides arredondadas e opostas, permitiram atribuir esse material ao grupo brasiliensis do gênero Holochilus, conforme Voss \& Carleton (1993), Pardiñas et al. (2013) e Gonçalves et al. (2015). Este grupo inclui duas espécies, H. brasiliensis (Desmarest, 1819) e H. vulpinus (Brants, 1827), cujas características diagnósticas não estão bem definidas e ambas possuem distribuição atual para o Rio Grande do Sul (Gonçalves et al., 2015). Desta forma, os fósseis foram identificados como Holochilus cf. H. brasiliensis.

\section{Oligoryzomys Bangs, 1900}

Espécie-tipo. Oryzomys navus Bangs, 1899 (= Oryzomys delicatus J.A. Allen \& Chapman, 1897).

\section{Oligoryzomys sp.}

(Figuras 5B, F)

Material. Dezessete fragmentos de maxilares e 69 fragmentos de dentários (Apêndice 1).

Descrição. O dentário é pequeno e a projeção capsular bem desenvolvida. Os molares superiores e inferiores são pequenos e as séries dentárias paralelas entre si. Os molares superiores possuem anterolofo desenvolvido. O anterocone do M1 é largo e possui um flexo anteromediano profundo, porém não tanto quanto em Juliomys. O M1 e M2 possuem uma ponte de esmalte ligando o paracone ao protocone e um mesolofo desenvolvido fusionado ao mesoestilo. O M3 possui hipoflexo raso. $\mathrm{O} \mathrm{m} 1$ apresenta cíngulo anterolabial, anterolofídeo, e mesolofídeo fusionado ao mesoestilídeo. O ectoestilídeo está presente nos molares inferiores de alguns exemplares, mas não há ectolofídeo, diferentemente de Juliomys.

Comentários. Essas características e a ausência de protoestilo e de ectolofídeo permitiram atribuir o material ao gênero Oligoryzomys, conforme Weksler \& Bonvicino (2015). Dentre as 19 espécies atualmente reconhecidas, duas ocorrem no Rio Grande do Sul, O. flavescens (Waterhouse, 1837) e O. nigripes (Olfers, 1818), as quais podem ser em parte diferenciadas pelo comprimento das séries molares superiores $(\mathrm{CM} 1-\mathrm{M} 3=3,01$ a $3,58 \mathrm{~mm}$ e CM1-M3 = 3,06 a 4,28 mm, respectivamente; Machado et al., 2011) e inferiores $(\mathrm{Cm} 1-\mathrm{m} 3=3,1$ a $3,38 \mathrm{~mm}$ e Cm1-m3 = 3,38 a 3,76 mm, respectivamente; conforme medidas de espécimes recentes). Os valores obtidos para nove espécimes do sítio Garivaldino (Tabela 3) são maiores que os de $O$. flavescens e coincidem com aqueles de $O$. nigripes. No entanto, seria prematuro identificar a espécie do sítio Garivaldino apenas com base na distribuição atual e nessa característica.

Pseudoryzomys Hershkovitz, 1962

Espécie-tipo. Oryzomys wavrini Thomas, 1921 (= Hesperomys simplex Winge, 1887).

$$
\text { Pseudoryzomys simplex (Winge, 1887) }
$$

(Figuras 5C, G)

Material. 23 fragmentos de maxilares e 34 fragmentos de dentários (Apêndice 1).

Descrição. O maxilar e dentário possuem tamanho médio. Os molares superiores possuem cúspides opostas umas às outras. $\mathrm{O} \mathrm{M} 1$ possui anterocone indiviso. $\mathrm{O} \mathrm{M} 1 \mathrm{e} \mathrm{M} 2$ apresentam mesolofo incipiente. O M2 e M3 possuem anterolofo bem desenvolvido. O M1 apresenta quatro raízes e o M2 e o M3, três. Nos molares inferiores as cúspides são alternadas. $\mathrm{O} \mathrm{m} 1$ possui anteroconídeo indiviso com um fossetídeo central bem desenvolvido. $\mathrm{O} \mathrm{m} 1$ e o $\mathrm{m} 2$ possuem posterolofídeo bem desenvolvido. $\mathrm{O} \mathrm{m} 1$ possui quatro raízes e o $\mathrm{m} 2$ e $\mathrm{m} 3$, três.

Comentários. As características acima e a ausência de mesolofídeo nos molares inferiores permitiram atribuir o material a Pseudoryzomys simplex, única espécie reconhecida para o gênero conforme Voss \& Myers (1991).

\section{Sooretamys Weksler, Percequillo \& Voss, 2006}

Espécie-tipo. Mus angouya G. Fischer, 1814.

$$
\text { Sooretamys angouya (Fischer, 1814) }
$$

(Figuras 5D, H)

Material. Nove fragmentos de maxilares e quatro fragmentos de dentários (Apêndice 1).

Descrição. O maxilar e dentário são grandes e robustos. Os molares superiores e inferiores são grandes, cristados e 
pentalofodontes. Nos molares superiores o posterolofo é bem desenvolvido e conectado ao metacone por um metalófulo. $\mathrm{O}$ M1 possui anterocone indiviso e anterolofo desenvolvido e fusionado ao anteroestilo. No M1 e M2 há um muro mediano ligando o paracone ao protocone e o mesolofo é desenvolvido e conectado a um mesoestilo. O M2 possui protoflexo desenvolvido e duas fossetas internas. O M1 possui quatro raízes, sendo a raiz acessória labial de desenvolvimento variável, e o M2 e o M3 possuem três raízes. $\mathrm{O} \mathrm{m} 1$ e $\mathrm{m} 2$ possuem mesolofídeo bem desenvolvido e fusionado ao mesoestilídeo e ectoestilídeo bem desenvolvido. $\mathrm{O} \mathrm{m} 1$ possui três raízes e o $\mathrm{m} 2$ e $\mathrm{m} 3$, duas raízes.

Comentários. As características descritas acima permitiram referir esses espécimes do sítio Garivaldino a Sooretamys angouya, de acordo com Chiquito et al. (2014).

Tribo PHYLLOTINI Vorontsov, 1959

Calomys Waterhouse, 1837

Espécie-tipo. Mus bimaculatus Waterhouse, 1837 (= Mus laucha $\mathrm{G}$. Fischer, 1814).

Calomys sp.

(Figuras 5E, I)

Material. Dois fragmentos de maxilares e 11 fragmentos de dentários (Apêndice 1).

Descrição. O maxilar e o dentário são delicados e pequenos. $\mathrm{O}$ dentário possui três alvéolos para o $\mathrm{m} 3$. Os molares superiores e inferiores são muito pequenos. O M1 possui flexo anteromediano deslocado lingualmente, e o cônulo anterolabial é maior que o anterolingual.

Comentários. Essas características e ausência de mesolofo e mesolofídeo permitiram atribuir o material do sítio Garivaldino ao gênero Calomys, conforme Pardiñas (1999) e Salazar-Bravo (2015). São reconhecidas atualmente 17 espécies de Calomys, duas das quais ocorrem no Rio Grande do Sul, C. laucha (G. Fischer, 1814) e C. tener (Winge, 1887) (Salazar-Bravo, 2015). No entanto, as espécies de Calomys são diferenciadas principalmente por características morfológicas externas e genéticas (Salazar-Bravo, 2015). Considerando a fragmentação do material, não foi possível referi-lo a uma espécie em particular.

\section{Tribo WIEDOMYINI Reig, 1980}

Wilfredomys Avila-Pires, 1960

Espécie-tipo. Thomasomys oenax Thomas, 1928.

Wilfredomys oenax (Thomas, 1928)

(Figuras 2C, F)

Material. Um fragmento de maxilar e seis fragmentos de dentários (Apêndice 1).
Descrição. Acrista massetérica é bem marcada, principalmente a inferior. A projeção capsular é pouco desenvolvida. Os molares superiores e inferiores são pentalofodontes e possuem as cúspides opostas umas às outras. $\mathrm{O}$ anterocone do M1 possui um flexo anteromediano profundo, deslocado lingualmente e orientado obliquamente, sendo o cônulo anterolingual significativamente menor que o anterolabial. Os molares superiores possuem mesolofo desenvolvido fusionado ao mesoestilo e ao paralófulo. Um ectoestílideo incipiente está presente no $\mathrm{m} 2$ de um único espécime (UNISC 1757-215), sendo este, variável em desenvolvimento no $\mathrm{m} 3$. $\mathrm{O} \mathrm{m} 2$ e $\mathrm{m} 3$ possuem mesolofídeo desenvolvido.

Comentários. As características descritas acima e a ausência de ectolofídeo permitiram atribuir o material do sítio Garivaldino a Wilfredomys oenax, de acordo com Percequillo et al. (2004), Brandão (2015) e González et al. (2015a). Em comparação com espécimes recentes de W. oenax coletados em Santa Catarina (UFSC 4999, 5000, 5001, 5018), o material fóssil não apresenta ectolofídeo e o ectoestilídeo está presente em $\mathrm{m} 2$ e $\mathrm{m} 3 \mathrm{com}$ grau de desenvolvimento variável, enquanto os recentes possuem ectolofídeo bem desenvolvido no $\mathrm{m} 1$ e o ectoestilídeo bem desenvolvido no m2 e m3. Vaz-Ferreira (1960) e Pine (1980) relataram diferenças entre características morfológicas externas e cranianas entre espécimes de $W$. oenax coletados no Uruguai e o espécime tipo (coletado no Rio Grande do Sul). Pine (1980) sugeriu a possibilidade de existirem duas subespécies de $W$. oenax. No entanto, a espécie é rara, com poucos exemplares depositados em coleções científicas, sendo assim difícil verificar a existência de diferenças morfológicas consistentes entre suas populações.

\section{CONSIDERAÇÕES FINAIS}

A assembleia de roedores sigmodontíneos do sítio Garivaldino é abundante e rica, incluindo 572 espécimes atribuídos a 250 indivíduos e 17 táxons. A tribo Akodontini é a melhor representada, com oito táxons $(\mathrm{MNI}=116)$, seguida pelas tribos Oryzomyini, quatro táxons ( $\mathrm{MNI}=66$ ), Phyllotini, um táxon (MNI=9) e Wiedomyini, um táxon $(\mathrm{MNI}=4)$. Além destes, a amostra também contém os gêneros incertae sedis Delomys e Juliomys (MNI=39) e 16 indivíduos identificados apenas a nível de subfamília devido ao grau de fragmentação. Um número pouco menor de táxons (15) foi registrado para o sítio Pilger, também situado na parte nordeste do Rio Grande do Sul, cerca de $23 \mathrm{~km}$ a leste do Garivaldino (Hadler et al., 2016). Naquele sítio, diferentemente do Garivaldino, foi registrado predomínio da tribo Oryzomyini $(\mathrm{MNI}=67)$, representando $54,47 \%$ dos indivíduos.

Quatro táxons predominam no sítio Garivaldino e correspondem a mais de $50 \%$ dos indivíduos da amostra: Akodon sp. (19,2\%), Oligoryzomys sp. (15,2\%), Juliomys sp. $(11,2 \%)$ e Necromys lasiurus $(9,6 \%)$. Por outro lado, os sigmodontíneos predominantes no sítio Pilger foram: Pseudoryzomys simplex (43,09\% dos indivíduos), Necromys 
spp. (13,82\% dos indivíduos) e Gyldenstolpia sp. (8,94\% dos indivíduos) (Hadler et al., 2016).

O registro de Necromys obscurus no sítio Garivaldino representa o primeiro para o Quaternário do Brasil, e Thaptomys nigrita e Wilfredomys oenax são identificados pela primeira vez para o Holoceno do Rio Grande do Sul. T. nigrita possui registro prévio para o Quaternário da Serra da Abelha, Lagoa Santa, Minas Gerais (Winge, 1887). W. oenax foi mencionado para o Quaternário do Abismo Iguatemi, São Paulo, por Castro \& Langer (2011), no entanto os materiais figurados deixam dúvidas quanto à identificação e necessitam ser revisados.

Em função da proximidade espaço-temporal, pode ser feita uma comparação entre os 11 níveis inferiores do sítio Pilger (ver Hadler et al., 2016) e os 19 níveis superiores do sítio Garivaldino, referentes ao Holoceno inferior e médio. Os gêneros Bibimys, Delomys, Holochilus, Juliomys, Kunsia, Oxymycterus e Wilfredomys são exclusivos do Garivaldino, que inclui ainda todos os gêneros registrados nos níveis inferiores do Pilger. Dessa forma, no Holoceno inferior e médio no sítio Garivaldino encontram-se mais táxons de áreas florestais, como Delomys e Wilfredomys, e Juliomys é mais frequente do que Pseudoryzomys, situação contrária ao encontrado no sítio Pilger, sugerindo a existência de um ambiente mais florestal no primeiro caso no Holoceno inferior. Estas variações nas assembleias de escala local, considerando o raio de predação de uma coruja, são importantes, pois refletem a necessidade de ampla amostra de assembleias estudadas para fazer uma abordagem adequada da reconstrução paleoambiental.

Alguns táxons típicos de ambientes abertos, como, por exemplo, Necromys obscurus, Gyldenstolpia sp., Kunsia tomentosus e Pseudoryzomys simplex, registrados nos sítios Garivaldino e Pilger, não possuem registro atual para o Rio Grande do Sul. O primeiro ocorre mais ao sul, no Uruguai e Argentina, e os três últimos mais ao norte, em áreas de Cerrado (Figura 6). Outro exemplo de extinção regional é Thylamys sp., um marsupial didelfídeo, registrado para o sítio Pilger (Hadler et al. 2009). Além disso, Hadler et al. (2008) registraram a extinção de fundo de três espécies de roedores caviomorfos: Euryzygomatomys mordax, Dicolpomys fossor e Clyomys riograndensis, também associadas a ambientes abertos.

A comparação da assembleia de sigmodontíneos do sítio Garivaldino com o Quaternário de outras regiões brasileiras é limitada. O sítio Garivaldino está inserido no Holoceno inferior-médio e provém de um contexto arqueológico, enquanto os demais sítios são encontrados em ambientes cavernícolas, sem controle estratigráfico preciso e, muitas vezes, sem datação, sendo associados ao Pleistoceno superior/Holoceno inferior. Além disso, o material de alguns estados necessita revisão taxonômica (ver o indicado acima para o Abismo Iguatemi). Não obstante, observa-se maior similaridade taxonômica da assembleia de sigmodontíneos do sítio Garivaldino com aquelas do Mato Grosso do Sul (e.g. Akodon sp., Calomys sp. e Kunsia tomentosus; Salles et al., 2006) e de Minas Gerais (e.g. Calomys sp., Holochilus sp. e Oxymycterus sp.; Lund, 1840; Winge, 1887). Por outro lado, no Quaternário de São Paulo, Goiás, Bahia e Piauí são encontrados táxons distintos (e.g. Blarinomys breviceps, Hylaeamys megacephalus; Castro \& Langer, 2011).

Uma análise prévia dos atributos tafonômicos dos sigmodontíneos do sítio Garivaldino permitiu identificar intensa ação de raízes sobre os ossos e dentes. Estas marcas ocorrem em toda a superfície dos ossos e se diferenciam de marcas de digestão, que são mais pontuais (Fernandez-Jalvo \& Andrews, 1992). A presença de abundantes marcas de raízes poderia indicar uma exposição do material na superfície por tempo suficiente para ser recoberto por vegetação, talvez coincidindo com os períodos de abandono do sítio pelas populações humanas. Além disso, não foram observadas marcas de corrosão severa por digestão, estando o material condizente, numa primeira aproximação, com o grau de

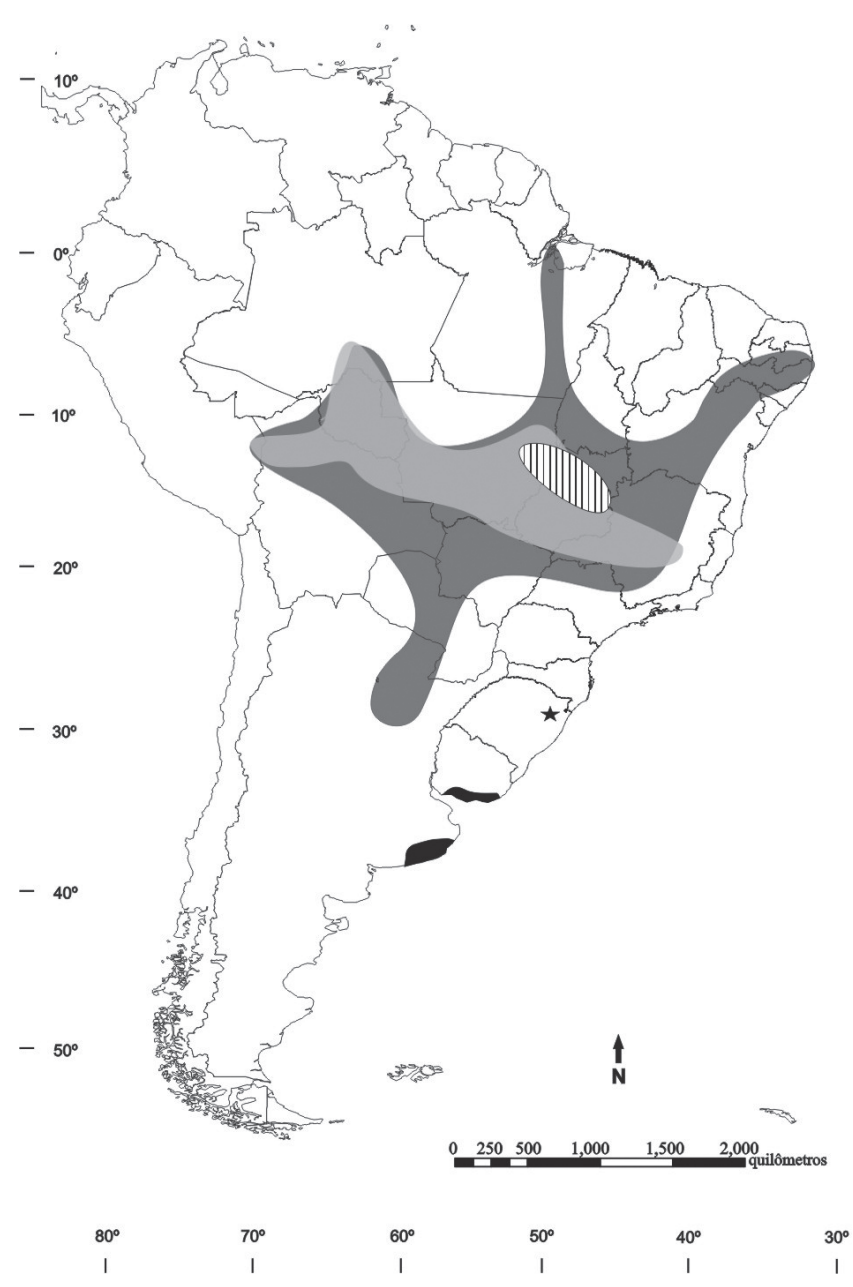

Figura 6. Distribuição geográfica atual de Necromys obscurus (preto); Pseudoryzomys simplex (cinza escuro); Kunsia tomentosus (cinza claro); Gyldenstolpia spp. (hachurado). A estrela indica a localização do sítio Garivaldino. Distribuições segundo Patton et al. (2015).

Figure 6. Current geographical distribution of Necromys obscurus (black); Pseudoryzomys simplex (dark gray); Kunsia tomentosus (light gray); Gyldenstolpia spp. (hatched). The star shows the location of Garivaldino site. Distributions according to Patton et al. (2015). 
digestão apresentado por corujas, como Tyto alba (Scopoli, 1769) (Andrews, 1990). No entanto, não é possível afirmar que todos os táxons tenham ingressado no sítio através de um único mecanismo. Kunsia e Gyldenstolpia, por exemplo, pelo grande tamanho corporal, poderiam servir como recurso potencial aos grupos humanos. Por outra parte, o material apresentou elevada fragmentação, restando na maioria das vezes apenas o corpo do dentário, como esperado para assembleias fósseis. A fragmentação e elevado número de dentários em relação aos maxilares podem sugerir ação de trampling sobre a assembleia (Andrews, 1990).

Análises tafonômicas e paleoambientais mais detalhadas para o entorno deste e de outros sítios (e.g. sítio Sangão) localizados no nordeste do Rio Grande do Sul estão sendo realizadas e deverão fornecer um panorama mais abrangente do ambiente e suas assembleias de micromamíferos no Holoceno do Sul do Brasil.

\section{AGRADECIMENTOS}

Os autores agradecem a S. Klamt pelo empréstimo do material do sítio Garivaldino; a P.C. Simões-Lopes e M.E. Graipel pelo acesso ao material da UFSC; a D. Voglino pela elaboração da Figura 1; a M. Gomes pelo auxílio na edição das fotografias; e a F.J. Fernández e um revisor anônimo pelas críticas e comentários ao artigo. Ao Laboratório Central de Microscopia Eletrônica da UFSC pelas fotografias em MEV. Ao CNPq (CNPq 444508/2014-7) (PH) e à Agencia Nacional de Promoción Científica y Tecnológica, Argentina (PICT 2014, \#1039) (UFJP) pelos auxílios concedidos.

\section{REFERÊNCIAS}

Anderson, S. \& Olds, N. 1989. Notes on Bolivian mammals. 5. Taxonomy and distribution of Bolomys (Muridae, Rodentia). American Museum Novitates, 2935:1-22.

Andrews, P. 1990. Owls, Caves and Fossils: predation, preservation and accumulation of small mammal bones in caves, with an analysis of the Pleistocene cave faunas from Westbury-SubMendip, Somerset, U.K. Chicago, The University of Chicago Press, $231 \mathrm{p}$.

Beisaw, A.M. 2013. Identifying and Interpreting Animal Bones: a manual. $1^{\text {a }}$ ed. College Station, Texas A \& M University Press, $214 \mathrm{p}$.

Bezerra, A.M.R. \& Pardiñas, U.F.J. 2016. Kunsia tomentosus (Rodentia: Cricetidae). Mammalian Species, 48:1-9. doi:10.1093/mspecies/sev013

Brandão, M.V. 2015. The presence of Wilfredomys oenax (Rodentia: Cricetidae) in São Paulo state, southeastern Brazil: a locally extinct species? Papéis Avulsos de Zoologia, 55:69-80. doi:10.1590/0031-1049.2015.55.04

Carleton, M.D. \& Musser, G.G. 1989. Systematic studies of Oryzomyine rodents (Muridae, Sigmodontinae): a synopsis of Microryzomys. Bulletin of the American Museum of Natural History, 191:1-83.

Castro, M.C. \& Langer, M.C. 2011. The mammalian fauna of Abismo Iguatemi, southeastern Brazil. Journal of Cave and Karst Studies, 73:83-92. doi:10.4311/jcks2010pa0140
Chiquito, E.A.; D’Elía, G. \& Percequillo, A.R. 2014. Taxonomic review of genus Sooretamys Weksler, Percequillo \& Voss (Rodentia: Cricetidae: Sigmodontinae): an integrative approach. Zoological Journal of the Linnean Society, 171:842-877. doi:10.1111/zoj.12146

Christoff, A.U.; Fagundes, V.; Sbalqueiro, I.J.; Mattevi, M.S. \& Yonegaga-Yassuda, Y. 2000. Description of a new species of Akodon (Rodentia: Sigmodontinae) from Southern Brazil. Journal of Mammalogy, 81:838-851.

Christoff, A.U.; Vieira, E.M.; Oliveira, L.R.; Gonçalves, J.W.; Valiati, V.H. \& Tomasi, P.S. 2016. A new species of Juliomys (Rodentia, Cricetidae, Sigmodontinae) from the Atlantic Forest of Southern Brazil. Journal of Mammalogy, 97: 1469-1482. doi:10.1093/ jmammal/gyw082

Costa, L.P.; Pavan, S.E.; Leite, Y.L.R. \& Fagundes, V. 2007. A new species of Juliomys (Mammalia: Rodentia: Cricetidae) from the Atlantic forest of southeastern Brazil. Zootaxa, 1463:21-37. doi:10.5281/zenodo. 176477

D’Elía, G.; Pardiñas, U.F.J. \& Myers, P. 2006. An introduction to the genus Bibimys (Rodentia: Sigmodontinae): phylogenetic position and alpha taxonomy. In: E. Lacey (ed.) Mammalian Diversification: from Chromosomes to Phylogeography, University of California Press, p. 147-182.

Fernandez-Jalvo, Y. \& Andrews, P. 1992. Small mammal taphonomy of Gran Dolina, Atapuerca (Burgos), Spain. Journal of Archaeological Science, 19:407-428. doi:10.1016/03054403(92)90058-B

Galliari, C.A. \& Pardiñas, U.F.J. 2000. Taxonomy and distribution of the sigmodontine rodents of genus Necromys in central Argentina and Uruguay. Acta Theriologica, 45:211-232.

Gonçalves, P.R. \& Oliveira, J.A. 2014. An integrative appraisal of the diversification in the Atlantic forest genus Delomys (Rodentia: Cricetidae: Sigmodontinae) with the description of a new species. Zootaxa, 3760:1-38. doi:10.11646/ zootaxa.3760.1.1

Gonçalves, P.R.; Teta, P. \& Bonvicino, C. 2015. Genus Holochilus Brandt, 1835. In: J.L. Patton; U.F.J. Pardiñas \& G. D’Elía (eds.) Mammals of South America, vol. 2, University of Chicago Press, p. $325-335$.

González, E.M. 2000. Un nuevo género de roedor sigmodontino de Argentina y Brasil (Mammalia: Rodentia: Sigmodontinae). Comunicaciones Zoológicas del Museo de Historia Natural de Montevideo, 12:1-12.

González, E.M.; Oliveira, J.A. \& Pardiñas, U.F.J. 2015a. Genus Wilfredomys Avila-Pires, 1960. In: J.L. Patton; U.F.J. Pardiñas \& G. D'Elía (eds.) Mammals of South America, vol. 2, University of Chicago Press, p. 105-107.

González, E.M.; Oliveira, J.A. \& Teta, P. 2015b. Genus Juliomys E.M. González, 2000. In: J.L. Patton; U.F.J. Pardiñas \& G. D'Elía (eds.) Mammals of South America, vol. 2, University of Chicago Press, p. 92-96.

Hadler, P.; Cherem, J.J.; Turbay, R.; Alberti, A. \& Pardiñas, U.F.J. 2016. Diversidade de pequenos mamíferos (Didelphimorphia e Rodentia) do Holoceno do Nordeste do Estado do Rio Grande do Sul, Brasil: implicações taxonômicas e paleoambientais. Revista Brasileira de Paleontologia, 19:126-144. doi:10.4072/ rbp.2016.1.10

Hadler, P.; Dias, A.S. \& Bauermann, S.G. 2013. Multidisciplinary studies of Southern Brazil Holocene: archaeological, palynological and paleontological data. Quaternary International, 305:119-126. doi:10.1016/j.quaint.2012.09.026 
Hadler, P.; Goin, F.J.; Ferigolo, J. \& Ribeiro, A.M. 2009. Environmental change and marsupial assemblages in Holocene successions of Southern Brazil. Mammalian Biology, 74:87-99. doi:10.1016/j.mambio.2008.03.003

Hadler, P.; Verzi, D.H.; Vucetich, M.G.; Ferigolo, J. \& Ribeiro, A.M. 2008. Caviomorphs (Mammalia, Rodentia) from the Holocene of Rio Grande do Sul State, Brazil: Systematics and Paleoenvironmental Context. Revista Brasileira de Paleontologia, 11:97-116. doi:10.4072/rbp.2008.2.03

Hershkovitz, P. 1955. South american marsh rats, genus Holochilus, with a summary of sigmodont rodents. Fieldiana: Zoology, 37:639-687. doi:10.5962/bhl.title.2902

Hershkovitz, P. 1962. Evolution of Neotropical cricetine rodents (Muridae), with special reference to the Phyllotine group. Fieldiana: Zoology, 46:1-524. doi:10.5962/bhl.title.2781

Hershkovitz, P. 1990. Mice of the Akodon boliviensis size class (Sigmodontinae, Cricetidae), with the description of two new species from Brazil. Fieldiana:Zoology, 57:1-35. doi:10.5962/ bhl.title.3137

Hershkovitz, P. 1994. The description of a new species of South American Hocicudo, or Long-Nose Mouse, Genus Oxymycterus (Sigmodontinae, Muroidea), with a critical review of the generic content. Fieldiana: Zoology, 79:1-43. doi:10.5962/bhl.title.3351

Hershkovitz, P. 1998. Report on some sigmodontine rodents collected in southeastern Brazil with descriptions of a new genus and six new species. Bonner zoologische Beiträge, 47:193-256.

Hinojosa, F.; Anderson, S. \& Patton, J.L. 1987. Two new species of Oxymycterus (Rodentia) from Peru and Bolivia. American Museum Novitates, 2898:1-17.

Lund, P.W. 1840. Nouvelles recherches sur la faune fossile du Brésil (extraits d'une lettre adressée aux rédacteurs, et datée de Lagoa Santa, ler Avril 1840). Annales des Sciences Naturelles, Serie 2, 13:310-319.

Lund, P.W. 1950. Memórias sobre a Paleontologia Brasileira. Rio de Janeiro, Instituto Nacional do Livro, 552 p. (Notas revistas e comentadas por Carlos de Paula Couto)

Machado, L.F.; Paresque, R. \& Christoff, A.U. 2011. Anatomia comparada e morfometria de Oligoryzomys nigripes e $O$. flavescens (Rodentia, Sigmodontinae) no Rio Grande do Sul, Brasil. Papéis Avulsos de Zoologia, 51:29-47. doi:10.1590/ S0031-10492011000300001

Machado, L.F.; Passaia, M.H.; Rodrigues, F.P.; Peters, F.B.; Sponchiado, J.; Valiati, V.H. \& Christoff, A.U. 2015. Molecular phylogenetic position of endangered Wilfredomys within Sigmodontinae (Cricetidae) based on mitochondrial and nuclear DNA sequences and comments on Wiedomyini. Zootaxa, 3986:421-434. doi:10.11646/zootaxa.3986.4.2

Mentz-Ribeiro, P.A. \& Ribeiro, C.T. 1999. Escavações Arqueológicas no sítio RS-TQ-58, Montenegro, RS, Brasil. Série Documento, Arqueologia, 10:1-86.

Pardiñas, U.F.J. 1996. El registro fósil de Bibimys Massoia, 1979 (Rodentia) en la Argentina. Consideraciones sobre los Scapteromyini (Cricetidae, Sigmodontinae) y su distribución durante el Plioceno-Holoceno en la región pampeana. Mastozoología Neotropical, 3:15-38.

Pardiñas, U.F.J. 1999. Los roedores muroideos del Pleistoceno tardio-Holoceno en la región pampeana (sector este) y Patagonia (República Argentina): Aspectos taxonómicos, importância bioestratigráfica y significación paleoambiental. Universidad Nacional de La Plata, Tese de doutorado, 283 p.
Pardiñas, U.F.J.; D’Elía, G. \& Teta, P. 2008. Una introducción a los mayores sigmodontinos vivientes: revisión de Kunsia Hershkovitz, 1966 y descripción de un nuevo género (Rodentia: Cricetidae). Arquivos do Museu Nacional, 66:509-594.

Pardiñas, U.F.J.; D’Elía, G. \& Teta, P. 2015a. Genus Bibimys Massoia, 1979. In: J.L. Patton; U.F.J. Pardiñas \& G. D’Elía (eds.) Mammals of South America, vol. 2, University of Chicago Press, p. 204-208.

Pardiñas, U.F.J.; Teta, P.; Alvarado-Serrano, D.; Geise, L.; Jayat, J.P.; Ortiz, P.E.; Gonçalves, P.R. \& D’Elía, G. 2015b. Genus Akodon Meyen, 1833. In: J.L. Patton; U.F.J. Pardiñas \& G. D’Elía (eds.) Mammals of South America, vol. 2, University of Chicago Press, p. 144-204.

Pardiñas, U.F.J.; Teta, P.; Ortiz, P.E.; Jayat, J.P. \& Salazar-Bravo, J. 2015c. Genus Necromys Ameghino, 1889. In: J.L. Patton; U.F.J. Pardiñas \& G. D'Elía (eds.) Mammals of South America, vol. 2, University of Chicago Press, p. 232-247.

Pardiñas, U.F.J.; Teta, P.; Voglino, D. \& Fernández, F.J. 2013. Enlarging rodent diversity in west-central Argentina: a new species of the genus Holochilus (Cricetidae, Sigmodontinae). Journal of Mammalogy, 94:231-240. doi:10.1644/12 mamm-a-216

Patton, J.; Pardiñas, U.F.J. \& D’Elía, G. 2015. Mammals of South America, Vol. 2. Chicago, University of Chicago Press, 1336 p.

Percequillo, A.R. 2006. Guia para a nomenclatura e padronização da descrição da dentição nos roedores sigmodontíneos. Boletim da Sociedade Brasileira de Mastozoologia, 47:5-11.

Percequillo, A.R.; Gonçalves, P.R. \& Oliveira, J.A. 2004 The rediscovery of Rhagomys rufescens (Thomas, 1886), with a morphological redescription and comments on its systematic relationships based on morphological and molecular (cytochrome b) characters. Mammalian Biology, 69:238-257. doi:10.1078/1616-5047-00140

Pine, R.H. 1980. Notes on rodents of the genera Wiedomys and Thomasomys (including Wilfredomys). Mammalia, 44:195-202.

Reig, O.A. 1972. The Evolutionary History of the South American Cricetid Rodents. University of London, Tese de doutorado, $451 \mathrm{p}$.

Reig, O.A. 1977. A proposed unified nomenclature for the enamelled components of the molar teeth of the Cricetidae (Rodentia). Journal of Zoology, 181:227-241. doi:10.1111/j.1469-7998.1977. tb03238.x

Rodrigues, P.H. 2008. Didelphimorphia, Chiroptera e Rodentia (Mammalia) do Holoceno do Estado do Rio Grande do Sul, Brasil: Aspectos taxonomicos, paleoambientais e paleoclimáticos. Programa de Pós-Graduação em Geociências, Universidade Federal do Rio Grande do Sul, Tese de doutorado, $203 \mathrm{p}$.

Salazar-Bravo, J. 2015. Genus Calomys Waterhouse, 1837. In: J.L. Patton; U.F.J. Pardiñas \& G. D'Elía (eds.) Mammals of South America, vol. 2, University of Chicago Press, p. 481-507.

Salles, L.O.; Cartelle, C.; Guedes, P.G.; Boggiani, P.C.; Janoo, A. \& Russo, C.A.M. 2006. Quaternary mammals from Serra da Bodoquena, Mato Grosso do Sul, Brazil. Boletim do Museu Nacional, Zoologia, 521:1-12.

Smith, M.F. \& Patton, J.L. 2007. Molecular phylogenetics and diversification of South American grass mice, genus Akodon. In: D. Kelt; E. Lessa \& J. Salazar-Bravo (eds.) Studies in contemporary mammalian biology. Papers honoring the remarkable career of Oliver P. Pearson, 1915-2003, University of California Publications in Zoology, p. 827-858. 
Vaz-Ferreira, R. 1960. Nota sobre Cricetinae del Uruguay. Archivos de la Sociedad de Biología de Montevideo, 24:66-75.

Voss, R.S. 1993. A Revision of the Brazilian Muroid Rodent Genus Delomys with Remarks on "Thomasomyine" Characters. American Museum Novitates, 3073:02-44.

Voss, R.S. \& Carleton, M.D. 1993. A new genus for Hesperomys molitor Winge and Holochilus magnus Hershkovitz (Mammalia, Muridae) with an analysis of its phylogenetic relationships. American Museum Novitates, 3085:2-40.

Voss, R.S. \& Myers, P. 1991. Pseudoryzomys simplex (Rodentia: Muridae) and the significance of Lund's Collections from the
Caves of Lagoa Santa, Brazil. Bulletin of the American Museum of Natural History, 206:414-432.

Weksler, M. \& Bonvicino, C.R. 2015. Genus Oligoryzomys Bangs, 1900. In: J.L. Patton; U.F.J. Pardiñas \& G. D’Elía (eds.) Mammals of South America, vol. 2, University of Chicago Press, p. 417-437.

Winge, H. 1887. Jordfundne og nulevende Gnavere (Rodentia) fra Lagoa Santa, Minas Geraes, Brasilien. E Museo Lundii, 1:1-178.

Received in September, 2016; accepted in March, 2017. 
Apêndice 1. Lista do material estudado. Abreviações: Dn, dentário; Mx, maxilar; dir, direito; esq, esquerdo. Os dentes, se presentes, são indicados entre parênteses.

Appendix 1. List of the studied material. Abbreviations: Dn, dentary; Mx, maxilar; dir, right; esq, left. The teeth, if present, are indicated in brackets.

Sigmodontinae: UNISC 1753-218, 1753-258, 1753-296, 1754-235, 1755-217, 1756-232, 1756-233, 1756-236, 1757-213, 1757-243, 1758-226, 1760-202, 1761-208, 1765-211, 1766-207, Dn-esq; UNISC 1753-228, 1754-211, 1755-208, 1755-210, 1755-230, 1756-239, 1757-228, 1757-236, 1757-244, 1757252, 1758-238, 1761-216, 1762-204, 1774-200, Dn-dir; UNISC 1753-252, Dn-esq (m1); UNISC 1759-203, Mx-esq (M2); UNISC 1761-210, Dn-dir (m1); UNISC 1761-228, Mx-esq; UNISC 1770-205, Mx-dir. Delomys sp.: UNISC 1752-202, 1752-212, Dn-esq (m1-m3); UNISC 1752-220, 1756-216, Dn-dir (m1-m3); UNISC 1752-225, 1753-289, 1757-239, 1758-222, 1761-221, Dn-dir (m1-m2); UNISC 1753-215, 1754-210, 1754-221, Dn-esq (m1-m2); UNISC 1753-268, 1758-233, Dn-dir (m2); UNISC 1753-305, 1756-260, Mx-esq (M1-M3); UNISC 1753-312, 1753-317, 1756-252, Mx-dir (M1-M2); UNISC 1754-219, 1755-224, Dn-dir (m2-m3); UNISC 1754-231, 1756-235, Dn-esq (m2); UNISC 1755-241, 1755-245, 1758-209, Mx-dir (M1); UNISC 1756-257, 1758-210, Mx-esq (M1-M2); UNISC 1757-223, Dn-esq (m2-m3); UNISC 1761-220, Dn-esq (m1). Juliomys sp.: UNISC 1751-202, 1753-281, 1754-202, 1756-210, 1756-227, 1758-231, Dn-esq (m3); UNISC 1751-204, 1751-210, 1754-237, Dn-dir (m2); UNISC 1751-209, Dn-dir (m2-m3); UNISC 1752-203, 1757-265, 1755-247, Mx-dir (M1-M3); UNISC 1752-206, Mx-dir (M2-M3); UNISC 1752-209, 1753-216, 1753-249, 1753-250, 1754-212, 1755-233, Dn-esq (m1-m3); UNISC 1752-214, 1752-239, 1753-222, 1753-257, 1754-214, 1754-215, 1754-220, 1754-229, 1754-230, 1754-240, 1755213, 1756-230, 1756-240, 1761-213, Dn-dir (m1); UNISC 1752-217, 1753-201, 1753-240, 1754-222, 1755-206, 1755-223, 1756-218, 1762-203, Dn-esq (m1-m2); UNISC 1752-228, 1752-229, 1753-203, 1754-209, Dn-dir (m1-m3); UNISC 1752-235, 1757-210, Dn-dir (m1-m2); UNISC 1753-206, 1756206, Dn-dir (m3); UNISC 1753-233, 1755-216, 1759-209, Dn-esq (m1); UNISC 1753-244, Dn-esq (m2-m3); UNISC 1753-254, 1756-219, Dn-dir (m1 e m3); UNISC 1753-303, 1753-304, 1753-306, 1753-314, Mx-esq (M1-M3); UNISC 1753-308, 1764-203, Mx-dir (M1-M2); UNISC 1753-311, Mx-dir (M2); UNISC 1753-315, 1756-259, Mx-esq (M2-M3); UNISC 1753-320, 1756-253, Mx-dir (M1); UNISC 1756-215, Dn-esq (m2); UNISC 1757-264, Mx-esq (M1 e M3). Akodon sp.: UNISC 1751-201, 1753-274, 1754-239, 1755-200, 1755-215, 1766-206, Dn-dir (m1-m3); UNISC 1751-203, 1752-226, 1752-230, 1752-240, 1753-209, 1753-229, 1753-241, 1753-267, 1753-275, 1753-285, 1754-213, 1755-221, 1755-225, 1755-237, 1755-238, 1756-237, 1759-205, 1761-211, 1770-201, Dn-dir (m1-m2); UNISC 1751-205, 1756-211, 1757-245, 1758-241, Dn-dir (m2); UNISC 1751-206, 1752-222, 1752-232, 1752-233, 1752-237, 1753-276, 1753-278, 1753-282, 1755-211, 1755-228, 1757-241, 1757-249, 1758-218, 1759-208, 1761-225, 1766-209, Dn-dir (m1); UNISC 1752-218, 1753-210, 1753-242, 1753-248, 1753-251, 1753-286, 1754-216, 1755-232, 1755-236, 1756-214, 1756-241, 1756-244, 1757-208, 1757250, Dn-esq (m1-m2); UNISC 1752-221, Dn-esq (m2-m3); UNISC 1752-223, 1754-205, 1756-231, 1756-245, 1758-227, Dn-esq (m1-m3); UNISC 1753-204, 1753-213, 1753-226, 1753-238, 1753-259, 1753-270, 1753-271, 1753-273, 1753-284, 1754-227, 1754-238, 1755-214, 1755-218, 1755-231, 1755-235, 1756-212, 1756-220, 1756-224, 1756-229, 1756-238, 1758-232, 1761-218, 1762-202, Dn-esq (m1); UNISC 1753-217, 1753-224, 1753-265, 1754-241, 1755-229, Dn-esq (m2); UNISC 1753-292, 1756-213, Dn-dir (m1 e m3); UNISC 1753-301, 1756-249, 1757-266, Mx-dir (M1-M2); UNISC 1755-239, 1755-250, Mx-esq (M1-M2); UNISC 1757-271, Mx-esq (M2). Bibimys labiosus: UNISC 1752-213, 1753-255, Dn-dir (m1-m3); UNISC 1754-232, Dn-dir (m2); UNISC 1756-217, Dn-esq (m1-m2); UNISC 1756-247, Dn-dir (m1); UNISC 1757-232, 1774-201, Dn-esq; UNISC 1757-234, 1757-238, Dn-dir (m1-m2); UNISC 1758-207, Mx-dir (M1-M2); UNISC 1771-201, Dn-esq (m1-m3); UNISC 1771-202, Dn-esq (m1). Gyldenstolpia sp.: UNISC 1751-200, Dn-dir (m1 e m3); UNISC 1754-200, Dn-dir; UNISC 1754-206, Dn-dir (m2); UNISC 1756-200, 1760-204, Dn-dir (m1-m3); UNISC 1756-250, Mx-esq (M1); UNISC 1759-202, 1765-202, Mx-dir (M1-M2); UNISC 1765-200, 1770-203, Mx-esq (M1-M3); UNISC 1765-204, 1765-205, 1766-200, Dn-esq (m1-m3); UNISC 1768-201, Dn-esq (m2-m3); UNISC 1768-203, Dn-dir (m1-m2); UNISC 1769-209, Dn-esq (m1 e m3). Kunsia tomentosus: UNISC 1757-200, Mx-esq (M1-M3); UNISC 1761-200, Mx-dir (M1-M2); UNISC 1761-227, Mx-dir (M2-M3); UNISC 1767-203, Mx-esq (M1-M2); UNISC 1769-200, Dn-esq. Necromys lasiurus: UNISC 1751-208, Mx-esq (M2); UNISC 1752-211, 1753-225, 1753-234, 1755-207, 1756-228, 1756-242, 1757-218, 1757-254, Dn-esq (m1-m2); UNISC 1752-227, 1753-239, 1753-256, 1754-208, 1754-225, 1754-225, 1755-205, 1756204, 1757-231, 1757-247, 1757-253, 1758-228, 1761-215, 1763-203, 1765-208, 1767-202, Dn-dir (m1); UNISC 1753-212, 1753-290, 1756-209, 1757-222, 1761-219, Dn-dir (m1-m2); UNISC 1753-219, 1753-269, 1753-294, 1754-242, 1755-209, 1756-223, 1757-216, 1757-229, 1761-214, 1765-212, Dn-esq (m1); UNISC 1753-263, Dn-esq (m1 e m3); UNISC 1753-313, 1753-321, 1753-324, 1755-249, 1756-254, 1757-256, 1757-261, 1761-229, Mx-dir (M1M2); UNISC 1754-228, Dn-esq (m1-m3); UNISC 1754-243, Mx-dir (M1-M3); UNISC 1756-201, 1768-206, 1770-202, Dn-esq; UNISC 1756-243, Dn-esq (m2); UNISC 1756-255, 1756-256, Mx-dir (M1); UNISC 1757-224, Dn-dir (m2); UNISC 1757-255, 1759-200, 1761-204, Mx-esq (M1-M2); UNISC 1757-270, 1758-243, Mx-esq (M1); UNISC 1761-217, Dn-dir (m1 e m3); UNISC 1766-208, Dn-dir. Necromys obscurus: UNISC 1752-208, 1753-277, 1757-204, 1758-220, 1760-200, 1764-200, 1769-206, Dn-esq (m1-m2); UNISC 1752-215, 1753-214, 1754-217, 1756-205, 1756-208, 1756226, 1757-221, 1759-210, 1759-211, Dn-dir (m1-m2); UNISC 1752-234, 1753-231, 1756-202, 1757-207, 1757-240, Dn-dir (m1); UNISC 1753-220, 1757-226, 1758-214, 1758-216, 1768-205, Dn-esq (m1); UNISC 1754-224, Dn-dir (m1-m3); UNISC 1755-246, 1758-208, 1761-205, Mx-dir (M1-M2); UNISC 1756-222, 1757-203, Dn-dir (m2); UNISC 1757-235, Dn-esq (m2); UNISC 1757-262, Mx-esq (M1-M2); UNISC 1758-202, 1765-203, Mx-esq (M1); UNISC 1771-200, Dn-dir. Oxymycterus sp.: UNISC 1753-200, 1758-234, 1769-207, Dn-dir (m1); UNISC 1753-260, Dn-esq (m2); UNISC 1753291, 1756-203, 1767-208, Dn-esq (m1-m3); UNISC 1753-299, 1753-307, Mx-dir (M1); UNISC 1753-319, Mx-dir (M1-M2); UNISC 1753-322, Mx-dir (M2); UNISC 1754-218, 1758-236, Dn-esq; UNISC 1755-204, 1756-234, 1758-229, Dn-dir (m2-m3); UNISC 1757-220, Dn-esq (m1-m2); UNISC 1757-257, Mx-esq (M1); UNISC 1758-225, 1765-206, Dn-dir (m1-m2); UNISC 1766-201, Dn-dir (m3); UNISC 1768-207, Dn-esq (m1). Thaptomys nigrita: UNISC 1752-231, Dn-esq (m1-m2); UNISC 1752-238, Dn-esq (m2-m3); UNISC 1755-226, Dn-esq (m1). Holochilus cf. H. brasiliensis: UNISC 1758-200, Mx-esq (M1); UNISC 1758-204, Mx-esq (M2-M3). Oligoryzomys sp.: UNISC 1752-205, 1753-318, 1753-323, 1755-244, Mx-dir (M1-M3); UNISC 1751-207, 1752-224, 1753-297, 1754-236, 1755-227, 1757-214, 1757-242, 1761-223, 1765-213, Dn-dir (m1); UNISC 1752-219, 1752-236, 1753223, Dn-esq (m2); UNISC 1753-205, 1757-233, Dn-esq; UNISC 1753-207, 1753-243, 1753-280, 1757-219, 1760-203, 1770-200, Dn-dir (m1-m2); UNISC 1753-227, 1753-232, 1755-220, 1755-222, 1757-248, 1764-201, Dn-esq (m1-m3); UNISC 1753-230, 1753-246, 1753-266, 1753-279, 1753-288, 1754207, 1754-226, 1754-233, 1755-212, 1757-227, 1757-230, 1757-246, 1757-251, 1758-223, 1758-230, 1759-204, 1761-212, 1765-207, 1765-210, Dn-esq (m1); UNISC 1753-237, 1753-245, 1753-262, 1753-287, Dn-dir (m2-m3); UNISC 1753-247, 1753-272, 1755-234, 1761-224, 1763-200, Dn-dir (m1-m3); UNISC 1753-264, 1754-223, 1755-240, 1764-202, 1769-204, Dn-esq (m1-m2); UNISC 1753-283, 1753-298, 1754-204, Dn-esq (m3); UNISC 1753-293, 1756-225, 1757-225, Dn-dir (m1 e m3); UNISC 1753-300, Mx-dir (M2); UNISC 1753-309, 1755-248, 1758-211, 1758-213, 1762-201, Mx-esq (M1-M2); UNISC 1753-310, 1761-207, Mx-dir (M1-M2); UNISC 1754-245, 1757-267, 1758-212, 1760-206, Mx-esq (M1); UNISC 1761-209, Dn-dir (m2); UNISC 1763-202, Mx-esq (M1 e M3); UNISC 1763-204, 1767-200, 1767-201, Dn-dir. Pseudoryzomys simplex: UNISC 1752-204, 1753-302, 1757-258, 1758205, 1766-211, Mx-esq (M1-M3); UNISC 1752-210, 1754-203, 1766-205, 1766-210, 1767-205, Dn-esq (m3); UNISC 1752-216, 1757-206, 1757-212, 1757-237, Dn-dir (m1-m3); UNISC 1753-208, 1755-201, 1758-215, Dn-esq (m1-m3); UNISC 1753-221, 1753-236, Dn-esq (m2); UNISC 1753-235, 1755-203, Dn-esq (m1); UNISC 1753-261, Dn-esq; UNISC 1755-202, 1760-201, Dn-esq (m2-m3); UNISC 1755-242, Mx-esq (M2); UNISC 1755-243, 1756-248, 1758-206, 1761-203, Mx-esq (M1); UNISC 1756-246, Mx-dir (M3); UNISC 1756-251, 1757-269, Mx-esq; UNISC 1757-202, 1768-204, Dnesq (m1-m2); UNISC 1757-205, 1757-217, 1758-224, 1758-237, 1769-203, Dn-dir; UNISC 1757-209, 1757-211, 1758-240, Dn-dir (m1); UNISC 1757- 
259, Mx-esq (M2-M3); UNISC 1757-260, 1758-203, Mx-dir (M1-M2); UNISC 1757-268, Mx-esq (M2); UNISC 1759-201, Mx-dir (M2-M3); UNISC 1759-206, 1769-205, Dn-dir (m2-m3); UNISC 1761-201, 1762-200, 1769-210, Mx-dir (M1-M3); UNISC 1761-206, Mx-dir; UNISC 1761-226, Dn-dir (m2); UNISC 1766-204, Dn-esq (m1 e m3); UNISC 1767-206, Mx-dir (M1); UNISC 1768-202, Dn-dir (m3). Sooretamys angouya: UNISC 1752-200, 1754-244, Mx-dir (M1-M3); UNISC 1752-201, 1760-205, Mx-dir (M1-M2); UNISC 1752-207, Dn-dir (m1-m3); UNISC 1754-201, Dn-esq (m3); UNISC 1756-258, 1758-201, Mx-dir (M1); UNISC 1757-272, Mx-esq (M1-M2); UNISC 1758-219, Dn-esq (m1-m3); UNISC 1767-204, Mx-dir (M2-M3); UNISC 1767-207, Mx-dir (M2); UNISC 1768-200, Dn-dir (m1). Calomys sp.: UNISC 1753-211, 1753-295, 1759-207, 1761-222, Dn-dir (m1-m2); UNISC 1755-219, 1758-239, 1763-201, Dn-dir; UNISC 1756-221, 1758-217, Dn-esq (m1); UNISC 1757-263, 1765-201, Mx-esq (M1); UNISC 1758-235, 1758242, Dn-dir (m1). Wilfredomys oenax: UNISC 1756-207, Dn-esq (m2); UNISC 1757-201, Dn-dir (m3); UNISC 1757-215, Dn-dir (m1-m2); UNISC 1758-221, Dn-esq (m2-m3); UNISC 1761-202, Mx-esq (M1-M3); UNISC 1766-203, Dn-dir (m2); UNISC 1769-202, Dn-dir (m1-m3). 